\begin{tabular}{llll}
\hline & & \multicolumn{2}{c}{ Abbreviations \& Acronyms } \\
\hline RUL & Remaining Useful Life & I-ARR & Interval Valued Analytical Redundancy Rela- \\
& & & tions \\
EOL & End of Life & DPP & Degradation Progression Parameter \\
DM & Degradation Model & RMAD & Relative Median Absolute Deviation \\
BG & Bond Graph & $R A$ & Relative Accuracy \\
BG-LFT & Bond Graph in Linear Fractional Transfor- & $R M S E$ & Root Mean Square Error \\
& mation & SIR & Sampling Importance Resampling \\
PF & Particle Filters & ARR & Analytical Redundancy Relations \\
\hline
\end{tabular}

\section{Particle Filter Based Hybrid Prognostics for Health Monitoring of Uncertain Systems in Bond Graph Framework}

\author{
Jha. Mayank Shekhar ${ }^{1}$, Dauphin-Tanguy. $G^{2}$, and Ould-Bouamama. $B^{3}$ \\ ${ }^{1,2}$ Centre de Recherche en Informatique, Signal et Automatique de Lille (CRIStAL) UMR CNRS 9189, Ecole Centrale \\ de Lille, Cité Scientifique 59650 Villeneuve d'Ascq France \\ ${ }^{1}$ jha.mayank.jha@gmail.com \\ ${ }^{2}$ genevieve.dauphin-tanguy@ec-lille.fr \\ ${ }^{3}$ Centre de Recherche en Informatique, Signal et Automatique de Lille (CRIStAL) UMR CNRS 9189, Polytech Lille, \\ Université de Lille 1, Cité Scientifique 59650 Villeneuve d' Ascq, France \\ ${ }^{3}$ Belkacem.Ouldbouamama@polytech-lille.fr
}

\begin{abstract}
The paper's main objective is to address the problem of health monitoring of system parameters in Bond Graph (BG) modeling framework, by exploiting its structural and causal properties. The system in feedback control loop is considered globally uncertain and parametric uncertainty is modeled in interval form. The system parameter is undergoing degradation (prognostic candidate) and its degradation model is assumed to be known a priori. The detection of degradation commencement is done in a passive manner which involves interval valued robust adaptive thresholds over the nominal part of the uncertain BG-derived Interval Valued Analytical Redundancy Relations (I-ARRs). The latter forms an efficient diagnostic module. The prognostics problem is cast as joint state-parameter estimation problem, a hybrid prognostic approach, wherein the fault model is constructed by considering the statistical degradation model of the system parameter (prognostic candidate). The observation equation is constructed from nominal part of the I-ARR. Using Particle Filter (PF) algorithms; the estimation of state of health (state of prognostic candidate) and associated hidden time-varying degradation progression parameters is achieved in probabilistic terms. A simplified variance adaptation scheme is proposed. Associated uncertainties which arise out of noisy measurements, parametric degradation process, environmental conditions etc. are effectively managed by PF. This allows the production of effective predictions of the remaining useful life of the prognostic candidate with suitable confidence bounds. The effectiveness of the novel methodology is demonstrated through simulations and experiments on a mechatronic system.
\end{abstract}

Keywords: Prognostics, Bond Graph, Intervals, Particle Filter, Remaining Useful Life, Robust Fault Detection 


\begin{tabular}{|c|c|c|c|}
\hline \multicolumn{4}{|c|}{ Notations } \\
\hline$\theta$ & System parameter & $\gamma^{d}$ & $\begin{array}{l}\text { Degradation progression parameter associated to } \\
\theta^{d}\end{array}$ \\
\hline$\theta^{d}$ & $\begin{array}{l}\text { System parameter under degradation } \\
\text { (prognostic candidate) }\end{array}$ & $\gamma^{d^{*}}$ & True value of $\gamma^{d}$ \\
\hline$\theta_{n}^{d}$ & Nominal value of $\theta^{d}$ & $p$ & probability \\
\hline$\Delta \theta$ & Additive uncertainty on $\theta$ & $\sigma_{X}$ & Standard deviation value of random specie $X$ \\
\hline$\delta_{\theta}$ & Multiplicative uncertainty on $\theta$ & $\sigma_{X}^{2}$ & Variance of population values of $X$ \\
\hline$\left[\delta_{\theta}\right]$ & $\begin{array}{l}\text { Multiplicative uncertainty in interval } \\
\text { form, equivalent to }\left[\delta_{\theta}, \overline{\delta_{\theta}}\right]\end{array}$ & $N$ & Number of particles in PF \\
\hline$\left[w_{\theta}\right]$ & $\begin{array}{l}\text { Uncertain effort or flow brought by } \\
\text { interval uncertainty on } \theta \text {, to the sys- } \\
\text { tem. }\end{array}$ & $\mathrm{w}_{k}^{i}$ & Weight of $i^{\text {th }}$ particle at discrete time $k$ \\
\hline$r_{n}(t)$ & $\begin{array}{l}\text { Numerical evaluation of the nominal } \\
\text { part of I-ARR }\end{array}$ & $y^{d}$ & Measurement of prognostic candidate $\theta^{d}$ \\
\hline$\hat{X}$ & Estimated value of species $X$ & $w^{d}(t)$ & Noise associated with measurement of $\theta^{d}$ \\
\hline$[\underline{R}, \bar{R}]$ & Interval valued ARR (I-ARR) & $\xi^{d}$ & Normally distributed random walk noise for $\gamma^{d}$ \\
\hline $\boldsymbol{\Psi}_{2}$ & $\begin{array}{l}\text { Interval function (uncertain part of I- } \\
\text { ARR) }\end{array}$ & $P^{d}$ & $\begin{array}{l}\text { Proportional gain constant in variance adaptation } \\
\text { of } \xi^{d}\end{array}$ \\
\hline$\Psi_{1}$ & Point-valued nominal part of I-ARR & $\mathrm{v}^{\xi^{d}}$ & RMAD (spread) of $\xi^{d}$ \\
\hline$\Psi_{2}$ & $\begin{array}{l}\text { Interval function } \boldsymbol{\Psi}_{2} \text { with point val- } \\
\text { ued arguments }\end{array}$ & $\mathrm{v}^{\xi^{d *}}$ & $\begin{array}{l}\text { Reference RMAD (spread) involved in variance } \\
\text { adaptation scheme }\end{array}$ \\
\hline$b(t)$ & Numerical evaluation of $\Psi_{2}$ & {$\left[\gamma_{l}^{d^{*}}, \gamma_{u}^{d^{*}}\right]$} & Interval containing $\gamma^{d^{*}}$ \\
\hline$[B(t), \overline{B(t)}$ & Range of interval function $\boldsymbol{\Psi}_{2}$ & $\overline{\hat{\gamma}}_{k}^{d}$ & Moving average of mean estimations of $\gamma^{d}$ \\
\hline
\end{tabular}

\section{Introduction}

Health Monitoring aims at ensuring system safety, reliability and efficient functionality and deals with fault detection and prediction of the Remaining Useful Life (RUL) of the system in a holistic way. While the former two is mainly dealt by using a diagnostic module, the latter is performed by a prognostic module. The primary focus lies in scheduling the maintenance actions according to progression of the system to a time where it may be considered beyond the limits of certified functionalities [1]. Such a time-horizon of interest is termed as the End of Life (EOL) and the time remaining until that point is called RUL of the system [2,3]. Prognostics are focused on the study of fault (or damage) evolution and prediction of the RUL of the system/component. Accurate prediction of EOL/RUL enables efficient and optimal planning of the future maintenance actions, and renders the capability of assessing reliability of the system [4]. This leads to system/component's life extension by modification of the system demand, operating conditions, workload etc. [5].

The failures of most systems can be attributed to the degradation of a given component, subsystem or material with time, environmental and operational conditions etc. Such system components/sub-systems can be identified as the poten- 
tial prognostic candidates through Failure Modes, Mechanisms and Effect Analysis or through other ways [6]. The underlying physical degradation is usually captured by Degradation Model(s) (DM) that can be obtained based upon physics of degradation or statistical (experimental) modeling approach as described in Gebraeel et al.[7] and Guo et al.[8]. In cases where physics of degradation is not available or reliable, the respective DM can be obtained statistically by finding a mathematical model that best fits a given set of degradation data. In this context, commonly employed DMs to fit the data are of linear, logarithmic, power or exponential form [8]. For example, approximation of degradation model by a linear part and logarithmic/exponential part [9], employment of exponential fit growth models [10], log-linear model for current drain degradation process [11] and stochastic degradation model [12].

Prognostic approaches are broadly divided into three categories [3, 13]: model-based prognostics [14], data-driven prognostics $[15,16]$ and hybrid prognostics $[9,17]$. In model based approach, the degradation model is physics based and requires a detailed understanding of the underlying phenomenon [1]. Inadequate modeling information, variation in behavioral physics or environmental conditions, un-modeled/unclassifiable sources of noise etc., result in limiting its adequacy. Data-driven methods tend to learn the damage progression. However, they generalize damage progression over large sets of component population and remain unreliable in assessing the variability of damage progression trend from component to component in a population [1]. As such, they provide inferior results especially in absence of complete data and large unit to unit variations. Hybrid approaches on the other hand, benefit from the fusion of the advantages of the former two [9]. They employ physics or statistical based degradation models and use measured information to adapt the damage progression, accounting for un-modeled variations, environmental changes, external noise etc.

Prognostic approaches set as a joint state-parameter estimation problem [18], have been widely useful and may fall under hybrid approach wherein, the prediction of RUL is based on current estimate of damage state and state of damage propelling hidden parameters. Prediction of the RUL is obtained as probability distribution and accounts for the various uncertainties involved [18-21].

Choice of the filter for estimation and prediction process depends on the assumptions that can be made about the system, and desired performance [22]. Well-known Kalman filter, an optimal estimator for linear systems, has been used for prognostics in[23, 24]. Extended Kalman Filter (EKF) [25]or Unscented Kalman filter [26], may also be used for parameter estimation posing the problem as joint state-parameter estimation or as Expectation-Maximization problem [27] etc. However, they remain restricted to additive Gaussian noise. Also, EKF being sub-optimal diverges quickly if the initial estimate of state is significantly far from true value, or the model considered for estimation is not correct [10]. Comprehensive comparative studies of filters for prognostic purposes are found in [10, 22, 28].

Set in Monte-Carlo framework, Particle Filters (PF) or Sequential Monte Carlo methods [29] form a suitable filter choice in this context, as it can be applied to non-linear systems corrupted with non-Gaussian noises for which, optimal solutions may be unavailable or intractable. Recently, particle filters have been exploited voraciously for prognostic methods [30, 31]. Significant works include prediction of end of discharge and EOL in lithium-ion batteries [32], battery health monitoring [33], prediction of battery grid corrosion [34], estimation and prediction of crack growth [35-38], fuel cell prognostics [9], application to damage prognostics in pneumatic valve from the Space Shuttle cryogenic refueling system $[18,39]$, estimation-prediction of wear as concurrent damage problem in centrifugal pumps with a variance control algorithm [20], employment in distributed prognosis [21], exploring uncertainty management options for prognostics [40] etc. Particle filters attract considerable attention [41], owing to the ever growing efforts being made for enhancement of performances and computational efficiency, such as the use of correction loops[42], fixed-lag filters [43] and the recently proposed adaption of the degradation model with a kernel smoothing method [44]. Although a large amount of research 
exists in model based diagnostics and prognostics, very few promise the achievement/implementation of all key requirements in a common framework and the realization through a unified modeling paradigm [45, 46].

In this context, Bond Graph (BG) modeling technique becomes a very suitable tool to deal with dynamic systems, especially those that belong to multi-energetic domains. A very brief and non-exhaustive introduction is given here. BG is a topological modeling language, where the exchange of energy between the different components of a dynamic system is captured in a graphical form. The energy exchange link is called a bond and there are two generic power variables named effort $e$ and flow $f$, associated with every bond, such that $e \times f=$ Power. The set of elements $\{I, C, R\}$, model the system parameters/component where $I, C$, and $R$ are the inertial element, capacitance element and dissipation element respectively. The latter along with the elements $\{0,1, T F, G Y\}$ define the junction structure (global structure of the system) where $T F$ and $G Y$ are the transformer element and gyrator element respectively. Junction $\mathbf{0}$ (or 1) implies that all the connected bonds have same effort (or flow) and the sum of flows (or efforts) equals zero. For efficient simulation of the physical behavior of the system, the computational order of the variables ( $e$ and $f$ ) must be decided systematically. For that purpose, the cause and effect decisions are described by the notion of causality. Causality in BG models is depicted by a perpendicular stroke on a bond. It determines whether the flow for a bond is computed from the effort or vice versa. The end of the bond which receives the effort is represented by the perpendicular stroke at that end of the bond. If all of the energy storage elements in a model are in integral form, the system is in integral causality. The constitutive equations of $I$ and $C$ respectively, in integral causality $\operatorname{are}^{2}$ (linear case): $f(t)=(1 / I) \int e(t) d t$ and $e(t)=(1 / C) \int f(t) d t$. For diagnosis task , where the initial conditions are unknown in real processes, the BG model is constructed in preferred derivative causality where the term "preferred" implies "wherever it is possible" . Derivative causality dictates the constitutive equation of $I$ and $C$ respectively, to be as (linear case): $e(t)=I d(f(t)) / d t$ and $f(t)=C d(e(t)) / d t$. For a detailed introduction from the $a b$ initio and various related behavioral, structural and causal properties, the readers are referred to following works [47-49] and [50].

For uncertain dynamic systems too, BG has been used extensively for modeling purposes and development of supervision techniques. This includes Fault Detection and Isolation (FDI) of complex systems [51], highly non-linear and complex thermo-chemical systems [52], non-linear mechatronic systems [53], intelligent and autonomous systems [54-56], industrial chemical reactors [57], hybrid systems [58] etc. In BG framework, the FDI is mainly based upon Analytical Redundancy Relations (ARRs)[59, 60] or by usage of the algebraic observers [55, 61]. Specifically, for uncertain systems, BG in Linear Fractional Transformation (BG-LFT)[62, 63] has been widely implemented for robust diagnosis by generating adaptive thresholds with respect to parametric uncertainties[64-66]. Very recently in Jha et al. [67], the authors have proposed modeling of parametric uncertainties in interval form. Unlike BG-LFT, where the threshold limits are simply the summation of the absolute values of each of the induced uncertain effortlflow at the junction [64] leading to an overestimation of threshold bounds, the interval valued thresholds consider the sensitivity of each uncertain candidate to the respective residual. Even though there has been wide implementation of BG for robust diagnosis of complex processes [65], there have been very little efforts if none, towards the development/integration of prognostic techniques in BG framework.

The few motivations propelling the development of this work are:

- Initial steps towards system level prognostics in BG framework: There are many benefits of using BG, including but not limited to: systematic graphical representation of the governing differential equations, efficient decomposition of

2 For any non-linear function $\Theta_{X}$ with respect to BG element $X$, integral causality: $f(t)=\Theta_{I}\left(\int e(t) d t\right)$ and $e(t)=\Theta_{I}\left(\int f(t) d t\right)$; derivative causality: $e(t)=\Theta_{I}^{-1} d(f(t)) / d t$ and $f(t)=\Theta_{C}^{-1} d(e(t)) / d t$ 
large scale multi-energetic systems into subsystems based upon functionality, an efficient understanding of the underlying physics, explicit knowledge of cause-effect relationships, validated FDI techniques at global as well as local level etc. On the other hand, benefits of system level prognostics are many [68]. For almost all practical purposes, any plant (the dynamic system of interest) is a feedback closed loop system such that the system outputs follow a desired reference. As such, the system level prognostics present unique challenges in that incipient parametric degradation may progress unnoticed in presence of controller compensated system outputs, resulting in non-estimation of the same till the saturation limit of controller is reached. BG derived ARRs being sensitive to system parameters and control inputs can be exploited for the same at local component level while being in closed loop regime. Such a kind of BG enabled health monitoring, can be achieved in a unified framework at global system level.

- Very few and inefficient existing residual based prognostic approaches: Most of the previous residual based attempts in BG framework consider damage progression deterministic in nature, incapable of adapting to the current damage progression and hence, reliability of prediction is minimal [69-72]. Moreover, uncertainties associated with measurements, operating conditions, process noise etc. have not been taken into account. This results in prediction of RUL without any associated confidence bounds, rendering it virtually useless for industrial certification and critical applications $[73,74]$.

- Inclusion of the recently developed diagnostic methodology: Recently, [67, 75] proposed a methodology of modeling uncertainties in interval form and the subsequent diagnosis through interval valued thresholds. This generates a genuine interest in its inclusion in an appropriate health monitoring framework.

This paper's main objective is to address the problem of prognostics in BG modeling paradigm while the system is considered globally uncertain and parametric uncertainty is modeled in interval form. This is achieved by casting the problem as a joint state-parameter estimation problem, a hybrid prognostic approach, wherein the fault model is constructed by considering the statistical degradation model of the system parameter. The system parameter is known $a$ priori to be undergoing degradation. Measurements are obtained from BG derived residuals (evaluation of ARRs). Using PF algorithms, estimation of state of the system parameter under degradation (prognostic candidate) along with the associated unknown hidden time varying Degradation Progression Parameters(s) (DPPs) is achieved and tracked to obtain the state of damage in probabilistic terms which is used for prediction of RUL of the system with respect to that parameter.

After this section, Section 2 details a fault detection algorithm where parametric uncertainty is modeled in interval form and Interval valued ARRs (I-ARRs) are derived systematically. The latter lead to the development of Interval valued robust thresholds over the nominal point-valued part of the I-ARRs. In Section 3, a novel methodology is proposed which includes construction of fault model and a novel way of obtaining the observation equation from the concerned nominal residual. In Section 4, the state of prognostic candidate and associated DPPs are estimated from the nominal part of the IARRs, sensitive to the latter and control inputs, in Monte-Carlo framework using PF algorithms. A novel variance control algorithm is proposed which ensures a suitable adaptation of random walk noise variance, once convergence is achieved in the estimation process. Prediction of the RUL is achieved in PF framework. Section 5 provides various evaluation metrics employed. In Section 6, the methodology is demonstrated through simulation and various issues are highlighted. In Section 7 , the methodology is tested experimentally by variation of frictional torque on a mechatronic system and Section 8 draws the conclusions. The various novel contributions of the paper are listed as follows:

- Integration of BG modeling framework and Monte Carlo framework for estimation of state of health and prediction of RUL.

- Exploitation of nominal part of I-ARRs derived in (BG framework) for detection of degradation beginning and prognosis of incipient parametric degradation in Monte Carlo framework using PF. 
- Obtaining the observation equation from the nominal part of I-ARRs and construction of local fault model such that state of the prognostic candidate and RUL prediction is obtained while system outputs are feedback controlled or otherwise.

- Accounting the various noises associated with degradation process and nominal residual output, for estimation and RUL prediction.

- Proportional control type variance adaptation algorithm with novel feedback condition that ensures a sustained convergence with low estimation variance (spread).

Major assumptions are:

- Only the system parameters are considered uncertain. Sensors are considered non-faulty.

- The system parameter (prognostic candidate) that undergoes degradation is assumed to be known a priori. The issue of isolation or isolability of the faulty candidate is assumed resolved.

- $\quad \mathrm{DM}$ of the prognostic candidate is assumed to be known a priori.

- $\quad$ Single fault (degradation) hypothesis is followed for an I-ARR considered.

- $\quad$ Noise associated with measurements (residuals) is assumed additive and normally distributed Gaussian in nature.

\section{Bond Graph Based Uncertain System Modeling}

Nominal model of any deterministic physical system may be modeled in BG form, in preferred integral causality, with nominal system parameters composed of basic elements $\boldsymbol{\theta} \in\{\boldsymbol{C}, \boldsymbol{I}, \boldsymbol{R}, \boldsymbol{T Y}, \boldsymbol{G} \boldsymbol{Y}\}$ with $\boldsymbol{\theta} \in \mathbb{R}^{N_{\theta}}$. Sub-script $n$ denotes the nominal value of the parameters. The sensor vector is formed by $\mathbf{Y}(t) \in[\boldsymbol{D e}(t), \boldsymbol{D} \boldsymbol{f}(t)]^{T}$ with $\boldsymbol{D e}(t) \in \mathbb{R}^{N_{D e}}$ being effort sensor vector and $\boldsymbol{D} \boldsymbol{f}(t) \in \mathbb{R}^{N_{D f}}$ being the flow sensor vector. The control/input vector is formed by $\mathbf{U}(t) \in[\boldsymbol{S e}(t), \boldsymbol{S} \boldsymbol{f}(t)]^{T}$ with $\boldsymbol{S e}(t) \in \mathbb{R}^{N_{S e}}$ and $\boldsymbol{S f}(t) \in \mathbb{R}^{N_{S f}}$ being respectively the source of effort and source of flow vectors. There is no uncertainty considered on system input (actuator/load/control input). The global system is considered uncertain with system parameters in interval form $[\underline{\boldsymbol{\theta}}, \overline{\boldsymbol{\theta}}] \in \mathbb{R}^{N_{m}}$ where $N_{m} \leq N_{\theta}$. The system parameter $\theta$ is modeled in interval form as $\theta \in[\underline{\theta}, \bar{\theta}]$, where $\forall \theta \in[\underline{\theta}, \bar{\theta}] \Rightarrow \underline{\theta} \leq \theta \leq \bar{\theta}$ and $[\underline{\theta}, \bar{\theta}] \in[\underline{\boldsymbol{\theta}}, \overline{\boldsymbol{\theta}}]$. Then,

$[\underline{\theta}, \bar{\theta}]=\left[\theta_{n}-\Delta \theta_{l}, \theta_{n}+\Delta \theta_{u}\right]$

$[\underline{\theta}, \bar{\theta}]=\left[\theta_{n}, \theta_{n}\right]+\left[-\Delta \theta_{l}, \Delta \theta_{u}\right]$

Here, $\Delta \theta_{l} \geq 0$ and $\Delta \theta_{u} \geq 0$ are the additive uncertainty/deviation on the left and right sides, over the nominal value $\theta_{n}$. $\left[\theta_{n}, \theta_{n}\right]$ is a degenerate interval with equal upper and lower bounds. For any additive uncertainty $\Delta \theta$ over $\theta$, the multiplicative uncertainty is defined as: $\delta_{\theta}=\Delta \theta / \theta_{n}$. Multiplicative interval uncertainty $\left[\underline{\delta_{\theta}}, \overline{\delta_{\theta}}\right]$ is expressed as in (3) such that $\delta_{\theta} \in\left[\underline{\delta_{\theta}}, \overline{\delta_{\theta}}\right]$. Then, the uncertain $\theta$ may be expressed as shown in (4).

$\left[\underline{\delta_{\theta}}, \overline{\delta_{\theta}}\right]=\left[-\Delta \theta_{l} / \theta_{n}, \Delta \theta_{u} / \theta_{n}\right]$

$[\underline{\theta}, \bar{\theta}]=\theta_{n}+\left[\underline{\delta_{\theta}}, \overline{\delta_{\theta}}\right] \theta_{n}$

Interval valued parameters are represented on an uncertain BG closely following the BG-LFT representation details of which can be referred in [63]. 


\subsubsection{Uncertainty Modeling and Representation on BG}

Uncertain system parameters can be represented in interval form on an uncertain BG by decoupling the nominal parameter value $\theta_{n} \in\left\{\boldsymbol{C}_{n}, \boldsymbol{I}_{n}, \boldsymbol{R}_{n}, \boldsymbol{T Y _ { n }}, \boldsymbol{G} \boldsymbol{Y}_{n}\right\}$, from the uncertain interval part $\left[\delta_{\theta}\right] \theta_{n}$ where for the notational simplicity, $\left[\delta_{\theta}, \overline{\delta_{\theta}}\right] \cong\left[\delta_{\theta}\right]$. The additional uncertain effort (or flow) is brought-in at the junction 1 (or 0 ) by interval uncertainty $\left[\delta_{\theta_{i}}\right]$. It is represented on uncertain BG by a combination of: virtual effort (or flow) detectors $D e^{*}: z_{\theta}\left(\right.$ or $D f^{*}: z_{\theta}$ ) and fictitious source of effort input $M S e:\left[w_{\theta}\right]$ (or fictitious source of flow input $M S f:\left[w_{\theta}\right]$ ) (see (7) and Fig. 1 for illustration). In fact, the fictitious sources $M S e:\left[w_{\theta}\right]$ (or $M S f:\left[w_{\theta}\right]$ ) are added to represent the introduction of an additional uncertain effort (or uncertain flow) generated by the interval uncertainty on the system. The virtual detectors $D e^{*}$ (or $D f *$ ) are used to represent the information exchange/transfer. The star ' $*$ ' is added as super-script for distinguishing the fictitious detectors (signals) from the real ones. In general, symbol ':' is used alongside a generic BG element to indicate the value in its respective characteristic equation. For instance, (see Fig. 1b) $R: R_{n}$ indicates that the system component modeled as resistor $R$ has the resistance value of $R_{n}$ in its characteristic equation $e_{R}=R \cdot f_{R}$.

For pedagogical illustration, a resistor element $R$ in resistance (imposed flow) causality is considered.

- Nominal case (see Fig. 1a): The characteristic equation with parameter in nominal state (without any uncertainty) is expressed as:

$e_{R}=R \cdot f_{R}$

- Uncertain case (see Fig. 1b): With multiplicative interval uncertainty $\left[\delta_{R}, \overline{\delta_{R}}\right]$, the characteristic law is expressed as:

$\left[\underline{e_{R}}, \overline{e_{R}}\right]=[\underline{R}, \bar{R}] \cdot f_{R}=R_{n}\left(1+\left[\underline{\delta_{R}}, \overline{\delta_{R}}\right]\right) \cdot f_{R}$

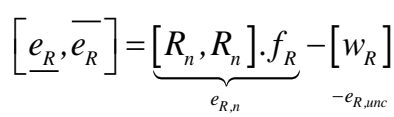

where $\left[w_{R}\right]=-\left[\underline{\delta_{R}}, \overline{\delta_{R}}\right] \cdot z_{R}=-\left[\underline{\delta_{R}}, \overline{\delta_{R}}\right] \cdot R_{n} \cdot f_{R}$. Interval valued uncertain effort $e_{R, \text { unc }}$ is brought at the $1-j u n c t i o n$ by $\left[w_{R}\right]$

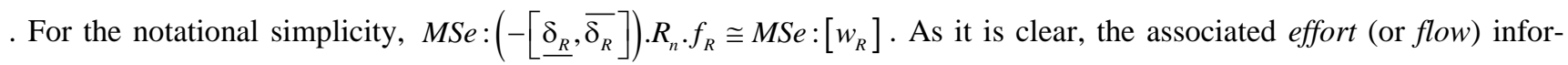
mation $\mathrm{z}_{R}=R_{n} \cdot f_{R}$, is brought to $M S e:\left[w_{R}\right]$ by the virtual detector $D e^{*}$.

For better illustration of the effort/flow transfer, Fig. 1c shows the equivalent block diagram representation of the uncertain BG in Fig. 1b.

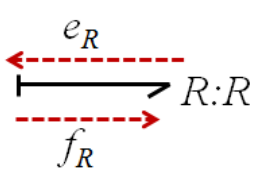

(a)

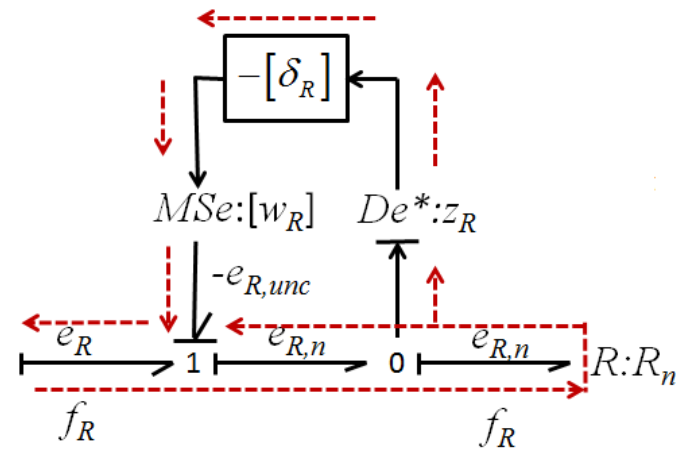

(b)

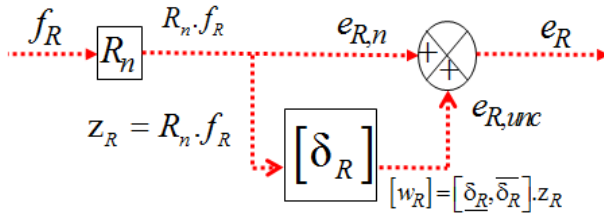

(c)

Fig.1. (a). Nominal $R$ element (resistance causality), (b) Uncertain $R$ element (resistance causality) in Interval form, (c) Equivalent Block Diagram Representation of Uncertain $R$ element (Illustration of Signal Transfer) 
Similarly, interval uncertainty can be modeled and represented for the other BG elements $I, C, G Y, T F, R S$ etc. For diagnosis based on ARR generation, the detectors are dualized such that effort detector De becomes a source of effort signal SSe and imposes the effort signal at the 0-junction connected to the detector. Flow detector Df becomes a source of flow signal SSf and imposes flow at the 1-junction connected to the detector [76].

\subsubsection{Interval Valued ARR Generation}

Classically, an ARR is a constraint relation derived from an over-constrained system/subsystem. It is expressed in terms of only known variables of the process [77]. For any function $\boldsymbol{f}$ and set of known variables $\boldsymbol{k}$, it has the form: $\boldsymbol{f}(\boldsymbol{k})=0$. In the context of BG modeling, an $A R R: f(\boldsymbol{S S e}(t), \boldsymbol{S S} \boldsymbol{f}(t), \boldsymbol{S e}(t), \boldsymbol{S} \boldsymbol{f}(t), \boldsymbol{\theta})=0$, where $\boldsymbol{\theta}$ is vector of system parameters. For deterministic systems, the properties and ARR generation algorithm are detailed in [59]. BG model in preferred derivative causality with dualized sensors are utilized to avoid unknown initial condition problem. For the uncertain systems, robust FDI is achieved by generation of uncertain ARRs with perfectly separable nominal part and uncertain part[64]. The aperate I-ARRs:

Step 2: Parametric uncertainties are modeled in interval form and represented on the nominal BG, as explained in section 2.1.1 to obtain uncertain BG.

Step 3: The candidate ARRs are generated from " 1 " or " 0 " junction, where power conservation equation dictates that sum of efforts or flows, respectively, is equal to zero, as shown in (8) and (9) with $s$ being the sign rendered to the bond due to energy convention.

- for 0 -junction:s $\sum s \cdot[\underline{f}, \bar{f}]+\sum S f+\sum_{i=0}^{i \leq N_{m}} s_{i} \cdot M S f:\left[w_{i}\right]=0$

- for 1 -junction: $\sum s \cdot[\underline{e}, \bar{e}]+\sum S e+\sum_{i=0}^{i \leq N_{m}} s_{i} \cdot M S e:\left[w_{i}\right]=0$

Step 4: The unknown effort or flow variables are eliminated using covering causal paths from unknown variables to known (measured) variables (dualized detectors), to obtain the I-ARRs, $[\underline{R}, \bar{R}]$ consisting of known variables only as shown in (10). The nominal part is characterized by point valued function $\Psi_{1}$, with point valued nominal parameters as coefficients of point valued measured variables (cf.(12)). It is separated from the interval valued part which is identified as an interval function $\boldsymbol{\Psi}_{2}$ (see Definition A.1 in Appendix A), sensitive to interval valued uncertainties (cf.(13)).

$[\underline{R}, \bar{R}]: \boldsymbol{\Psi}\left(\boldsymbol{\theta}_{n},[\underline{\boldsymbol{\theta}}, \overline{\boldsymbol{\theta}}],\left[w_{i}\right], \sum \operatorname{Se}, \sum \operatorname{Sf}, \boldsymbol{S S e}(t), \boldsymbol{S S f}(t)\right)$

$[\underline{R(t)}, \overline{R(t)}]: r_{n}(t)+[\underline{B(t)}, \overline{B(t)}]$

$r_{n}(t)=\Psi_{1}\left(\boldsymbol{\theta}_{\mathbf{n}}, \operatorname{SSe}(t), \operatorname{SSf}(t), \sum S e, \sum S f\right)$

$[\underline{B(t)}, \overline{B(t)}]=\boldsymbol{\Psi}_{2}\left([\underline{\boldsymbol{\theta}}, \overline{\boldsymbol{\theta}}],\left[\underline{\boldsymbol{\delta}_{\boldsymbol{\theta}}}, \overline{\boldsymbol{\delta}_{\boldsymbol{\theta}}}\right], \boldsymbol{S S e}(t), \boldsymbol{S S f}(t)\right)$

Hereafter, $r_{n}(t)$ being the numerical evaluation of the point-valued nominal part $\Psi_{1}$, will be referred to as nominal residual and $\boldsymbol{\Psi}_{2}$ will be termed as Uncertain Residual Interval Function (URIF). 
In BG framework, the DM of a system parameter under degradation $\theta^{d} \in \boldsymbol{\theta}, \boldsymbol{\theta} \in \mathbb{R}^{N_{\theta}}$ can be obtained from the time evolution profile of the respective ARR to which it is sensitive, assuming that the rest of the system parameters which are sensitive to the same, do not undergo any kind of progressive fault or degradation [69],[78]. Here, consider the point valued part of the $d^{\text {th }}$ I-ARR $r^{d}(t)$, such that with $\boldsymbol{\theta}^{\prime}=\boldsymbol{\theta} \backslash \theta^{d}(t), t>0, r_{n}^{d}(t) \neq 0$ :

$r^{d}(t)=\Psi_{1}^{d}\left(\theta^{d}(t), \boldsymbol{\theta}_{n}^{\prime}, \mathbf{S S e}(t), \mathbf{S S f}(t), \boldsymbol{S e}(t), \boldsymbol{S f}(t)\right)$

Here, the sub-script $n$ denotes nominal value. The computed values of $r^{d}(t)$ at time sample points give an implicit relation of the degradation profile of $\theta^{d}(t)$ in time. Assuming that implicit function theorem is satisfied [79], (14) gives a real valued function $\psi_{d}$ such that:

$\theta^{d}(t)=\psi_{d}\left(r^{d}(t), \boldsymbol{\theta}_{n}^{\prime}, \mathbf{S S e}(t), \mathbf{S S f}(t), \operatorname{Se}(t), \boldsymbol{S} \boldsymbol{f}(t)\right)$

Residual based DM should be obtained prior to prognostics i.e. prior to the phase when system's health monitoring is of interest.

\subsection{Fault Detection Using Interval Valued Thresholds}

In this work, the fault detection module is constructed for the robust detection of degradation commencement.

\subsubsection{Interval Valued Thresholds}

Consider point valued parametric deviation(s) $\delta_{\theta}$ such that $\forall[\underline{\theta}, \bar{\theta}] \in[\underline{\boldsymbol{\theta}}, \overline{\boldsymbol{\theta}}] \mid \theta(t) \in[\underline{\theta}, \bar{\theta}], \delta_{\theta} \in\left[\underline{\delta_{\theta}}, \overline{\delta_{\theta}}\right], b(t)$ is the numerical evaluation of function $\Psi_{2}$ with point valued arguments (cf.(16)).

$$
b(t)=\Psi_{2}\left(\delta_{\theta}, \theta(t), \operatorname{SSe}(t), \operatorname{SSf}(t)\right)
$$

$\Psi_{2}$ can be considered as the Natural Interval Extension Function of point valued function $\Psi_{2}$ (see Definition A.2 in Appendix A), with the point valued arguments and operators replaced by the corresponding interval arguments (timeinvariant here) and interval operators in the syntactic expression of the function $\Psi_{2}[80,81]$. Then, $\Psi_{2}$ can be expressed as finite sequence of interval arithmetic operations (evaluated as class code during implementation [81]) so that it is considered as a Rational Interval Function of $\Psi_{2}$ and hence, is inclusion isotonic (see Definition A.3 and Definition A.4 in Appendix A). Then, through Fundamental Theorem of Interval Analysis (see Theorem A.1 in Appendix A) the inclusion of (17) can be verified.

$b(t) \subseteq[\underline{B(t)}, \overline{B(t)}]$

274 Now, at all times, due to power conservation at the junction,

$r_{n}(t)+b(t)=0$

$\Rightarrow b(t)=-r_{n}(t)$

Thus, the change in effortflow brought by deviation at any instant in system is given by negative value of the nominal residual at that time. From (16),(17) and(18), following is used for fault detection:

Under nominal conditions:

$-r_{n}(t) \subseteq[\underline{B}, \bar{B}](t)$ 
$-r_{n}(t) \not \subset[\underline{B}, \bar{B}](t)$

\subsubsection{Robust Fault Detection}

The thresholds are generated by the range evaluation of URIF as shown in (19) and (20). In discrete time step $k$, the algorithm for fault detection is given in Table I. It should be noted that therein, the bounds of URIF $\boldsymbol{\Psi}_{2}$ is computed by expressing them as a sequence (computational graph or code list) of real valued functions [81].

\section{Fault model}

In this work, the system parameter that undergoes degradation is assumed to be known a priori. Let $\theta^{d}(t) \in \boldsymbol{\theta}$ be such a prognostic candidate. The objective in this paper is to estimate the state of $\theta^{d}(t)$ based upon information (measurement) provided by the values of nominal residual sensitive to $\theta^{d}(t), r_{n}^{d}(t)$.

\subsection{State Equation}

The parameter under degradation $\theta^{d}(t)$ is included as a tuple $\left(\theta^{d}, \gamma^{d}, g^{d}\right)$ to model the progressive fault where $g^{d}($.$) de-$ notes the linear/non-linear degradation progression function (DPF) obtained from the corresponding DM. The latter models the way degradation progresses in $\theta^{d}(t)$ :

$\theta^{d}(t)=g^{d}\left(\gamma^{d}(t), v^{\theta^{d}}(t)\right) ; \theta^{d}(t=0)=\theta_{n}^{d}$

where, $\gamma^{d}(t) \in \mathbb{R}^{N \gamma^{d}}$ is DPP vector and $\boldsymbol{v}^{\theta^{d}}(t) \in \mathbb{R}^{N_{v^{d}}}$ is the respective associated process noise vector. The fault model for $\left(\theta^{d}, \gamma^{d}, g^{d}\right)$ is constructed in state-space form by considering the parameter $\theta^{d}(t)$ as the state variable augmented with the DPP vector as,

$\dot{\boldsymbol{x}}^{d}(t)=\boldsymbol{f}^{d}\left(\boldsymbol{x}^{d}(t), \boldsymbol{v}^{x_{d}}(t)\right)$

where, $\boldsymbol{x}^{d}(t)=\left[\theta^{d}(t), \boldsymbol{\gamma}^{d}(t)\right]^{T}$ is the augmented state vector and $\boldsymbol{f}^{d}$ is state transition function following the Markovian assumption.

\subsection{Residual Based Observation Equation}

Here, the objective is to exploit the nominal residual for the estimation of state variables. This way, the nominal residual used for detection of degradation beginning can be further used furthur for estimation of state of health of the prognostic candidate and associated DPPs. This is possible if the ARR expression is altered to obtain the observation equation in an appropriate way, such that the nominal residual provides the measurements of state variables. For this purpose, a simple algebraic approach is proposed.

Theorem: Under the single degradation hypothesis, assuming the nominal part $r_{n}^{d}(t)$ of an I-ARR can be expressed as a linear combination of non-linear functions of $\theta^{d}(t)$, the measurement of the state $\theta^{d}(t)$ can be obtained from the negative value of $r_{n}^{d}(t)$.

Proof: Let $\theta^{d}(t)$ be the prognostic candidate and $\boldsymbol{\theta}^{\prime}=\boldsymbol{\theta} \backslash \theta^{d}(t)$. Assuming $r_{n}^{d}(t)$ can be expressed as,

$$
r_{n}^{d}(t)=\Xi\left(\boldsymbol{\theta}_{n}^{\prime}, \operatorname{SSe}(t), \boldsymbol{S S \boldsymbol { f }}(t), \boldsymbol{S e}(t), \boldsymbol{S} \boldsymbol{f}(t)\right)+\mathrm{A}^{T} \boldsymbol{\varphi}\left(\theta_{n}^{d}\right)
$$


where $\forall i \mid i=1,2 \ldots m, \mathrm{~A}^{m \times 1}=\left[a_{1} a_{2} \ldots a_{m}\right]^{T} \quad$ is $\quad$ a $\quad$ vector of known (measured system variables) with $a_{i}=\phi_{i}\left(\boldsymbol{\theta}_{n}{ }^{\prime}, \boldsymbol{S S e}(t), \boldsymbol{S S \boldsymbol { f }}(t), \boldsymbol{S e}(t), \boldsymbol{S} \boldsymbol{f}(t)\right)$ and $\boldsymbol{\varphi}^{m \times 1}\left(\theta^{d}(t)\right)=\left[\varphi_{1}\left(\theta^{d}(t)\right), \varphi_{2}\left(\theta^{d}(t)\right), \ldots . \varphi_{m}\left(\theta^{d}(t)\right)\right]^{T}$ is the vector of non-linear functions of $\theta^{d}(t)$. Then, $\forall t \geq 0$ power conservation at the BG junction where the corresponding I-ARR is derived, gives,

$$
r^{d}(t)=\Xi\left(\boldsymbol{\theta}_{n}^{\prime}, \operatorname{SSe}(t), \boldsymbol{S S \boldsymbol { f }}(t), \boldsymbol{S e}(t), \boldsymbol{S} \boldsymbol{f}(t)\right)+\mathrm{A}^{T} \boldsymbol{\varphi}\left(\theta^{d}(t)\right)=0
$$

or,

$$
\begin{aligned}
& r^{d}(t)=\Xi\left(\boldsymbol{\theta}_{n}^{\prime}, \mathbf{S S e}(t), \mathbf{S S f}(t), \sum S e, \sum S f,\right)+\mathrm{A}^{T} \boldsymbol{\varphi}\left(\theta_{n}^{d}\right)+\left(\mathrm{A}^{T} \boldsymbol{\varphi}\left(\theta^{d}(t)\right)-\mathrm{A}^{T} \boldsymbol{\varphi}\left(\theta_{n}^{d}\right)\right)=0 \\
& r^{d}(t)=r_{n}^{d}(t)+\mathrm{A}^{T}\left(\boldsymbol{\varphi}\left(\theta^{d}(t)\right)-\boldsymbol{\varphi}\left(\theta_{n}^{d}\right)\right)=0 \\
& \mathrm{~A}^{T}\left(\boldsymbol{\varphi}\left(\theta^{d}(t)\right)-\boldsymbol{\varphi}\left(\theta_{n}^{d}\right)\right)=-r_{n}^{d}(t)
\end{aligned}
$$

Thus, degradation state $\theta^{d}(t)$ can be linked implicitly to the measurements of $-r_{n}^{d}(t)$. Observation equation can be obtained as,

$$
y^{d}(t)=-r_{n}^{d}(t)=\mathrm{A}^{T}\left(\boldsymbol{\varphi}\left(\theta^{d}(t)\right)-\boldsymbol{\varphi}\left(\theta_{n}^{d}\right)\right)
$$

312 Corollary: When $\varphi\left(\theta_{n}^{d}\right)=\varphi\left(\theta_{n}^{d}\right)=\theta_{n}^{d}$, the vector $A=a_{1}, a_{1}=\phi_{1}\left(\boldsymbol{\theta}_{n}^{\prime}, \operatorname{SSe}(t), \operatorname{SSf}(t), \sum S e, \sum S f\right)$, can be understood as the coefficient function linking the fault value to the residual. It can be found as,

$$
a_{1}=\frac{\partial\left(r_{n}^{d}(t)\right)}{\partial\left(\theta^{d}(t)\right)}
$$

The observation equation argument in (26) includes known variables (sensor measurements, system parameters, inputs etc.) and their derivatives. It is heavily corrupted with noise, especially due to presence of derivative(s) of measured variables. In this work, the noise is considered additive, independent and identically distributed (i.i.d.) drawn from a zero mean normal distribution. It is assumed uncorrelated to $\boldsymbol{x}^{d}(t)$.Thus, from(26), observation equation is formed as,

$y^{d}(t)=h^{d}\left(\boldsymbol{x}^{d}(t)\right)+w^{d}(t)$

where $h^{d}($.$) is a nonlinear observation function obtained from (26)$ and $w^{d}(t) \sim \mathcal{N}\left(0, \sigma_{w^{d}}^{2}\right)$. The standard deviation $\sigma_{w^{d}}$, is approximated from residual measurements.

\section{Degradation Estimation And RUL Prediction}

321 In discrete time step $k \in \mathbb{N}^{*}$, the fault model $\left(\theta^{d}, \gamma^{d}, g^{d}\right)$ can be described in stochastic framework as,

$\boldsymbol{x}_{k}^{d}=\boldsymbol{f}_{k}^{d}\left(\boldsymbol{x}_{k-1}^{d}, \boldsymbol{v}_{k-1}^{\boldsymbol{x}_{d}}\right)$

$y_{k}^{d}=h^{d}\left(\boldsymbol{x}_{k}^{d}\right)+w_{k}^{d}$

where $\boldsymbol{x}_{k}^{d}=\left[\theta_{k}^{d}, \boldsymbol{\gamma}_{k}^{d}\right]^{T}, \boldsymbol{f}_{k}^{d}$ is state transition function (possibly non-linear) and is described by first order Markov model.

Measurements $y_{k}^{d}$ are assumed conditionally independent, given the state process $\boldsymbol{x}_{k}^{d}$. The likelihood function becomes as,

$$
p\left(y_{k}^{d} \mid \theta_{k}^{d}, \gamma_{k}^{d}\right)=\frac{1}{\sigma_{w_{k}^{d}} \sqrt{2 \pi}} \exp \left(-\left(y_{k}^{d}-h^{d}\left(x_{k}^{d}\right)\right)^{2} / 2 \sigma_{w_{k}^{d}}^{2}\right)
$$

With the beginning of degradation being detected by the FDI module as a fault at time step $k_{d}$, the prediction of 
problem in particle filter (PF) framework, where the estimation at time $k$ is obtained as probability density function (pdf) $p\left(\theta_{k}^{d}, \gamma_{k}^{d} \mid y_{k_{d}: k}^{d}\right)$, based upon history of measurements from the time of beginning of degradation $k_{d}$ up to $k, y_{k_{d}: k}^{d}$. In the following section, the method employed for degradation estimation and consequent prognostics is explained assuming that degradation begins at the start. In reality, information about $k_{d}$ will be given by fault detection module as described in Section 3.

\subsection{Degradation Estimation}

In this section, concise details about PF are provided. The related concepts mentioned here can be found detailed in [29] and [82]. The state distribution is approximated by set of discrete weighted samples or particles, $\left\{\left(\theta_{k}^{d, i}, \boldsymbol{\gamma}_{k}^{d, i}\right), \mathrm{w}_{k}^{i}\right\}_{i=1}^{N}$, where $N$ is the total number of particles and for $i^{\text {th }}$ particle at time $k, \theta_{k}^{d, i}$ is the estimate of the state (system faulty parameter here) and $\boldsymbol{\gamma}_{k}^{d, i}$ is the estimate of fault progression parameters. The weight associated with each particle is denoted by $\mathrm{w}_{k}^{i}$. The posterior density at any time step $k$ is approximated as,

$$
p\left(\theta_{k}^{d}, \gamma_{k}^{d} \mid y_{0: k}^{d}\right) \approx \sum_{i=1}^{N} \mathrm{w}_{k}^{i} \cdot \delta_{\left(\theta_{k}^{d}, \gamma_{k}^{d}\right)}\left(d \theta_{k}^{d} d \gamma_{k}^{d}\right)
$$

where $\delta_{\left(\theta_{k}^{d}, \gamma_{k}^{d}\right)}\left(d \theta_{k}^{d} d \gamma_{k}^{d}\right)$ denotes the Dirac delta function located at $\left(\theta_{k}^{d}, \gamma_{k}^{d}\right)$ and sum of the weights $\sum_{i=1}^{N} \mathrm{w}_{k}^{i}=1$.In this paper, sampling importance resampling (SIR) PF is employed for estimation of $p\left(\theta_{k}^{d}, \gamma_{k}^{d} \mid y_{0: k}^{d}\right)$, assuming that particles $\left\{\left(\theta_{k-1}^{d, i}, \gamma_{k-1}^{d, i}\right), \mathrm{w}_{k-1}^{i}\right\}_{i=1}^{N}$ are available as realizations of posterior probability $p\left(\theta_{k-1}^{d}, \gamma_{k-1}^{d} \mid y_{0: k-1}^{d}\right)$ at time $k-1$, with the following main steps:

- Realizations of prediction $p\left(\theta_{k}^{d}, \boldsymbol{\gamma}_{k}^{d} \mid y_{0: k-1}^{d}\right)$, is obtained in form of new set of particles $\left\{\left(\theta_{k}^{d, i}, \boldsymbol{\gamma}_{k}^{d, i}\right), \mathrm{w}_{k}^{i}\right\}_{i=1}^{N}$, with weights being chosen using the principle of importance sampling. The proposal importance density is chosen as the transitional prior $p\left(\boldsymbol{x}_{k}^{d, i} \mid \boldsymbol{x}_{k-1}^{d, i}\right)$, such that particles are generated by sampling from probability distribution of system noise $\boldsymbol{v}_{k-1}^{x_{d}}$ and simulation of the system dynamics of(29).

- Each sampled particle $\left(\theta_{k}^{d, i}, \boldsymbol{\gamma}_{k}^{d, i}\right)$ is then updated. The weight $\mathrm{w}_{k}^{i}$ is associated to each of the particles based on the likelihood of observation $y_{k}^{d}$ made at time $k$ as,

$$
\mathrm{w}_{k}^{i}=p\left(y_{k}^{d} \mid \theta_{k}^{d, i}, \boldsymbol{\gamma}_{k}^{d, i}\right) / \sum_{j=1}^{N} p\left(y_{k}^{d} \mid \theta_{k}^{d, j}, \boldsymbol{\gamma}_{k}^{d, j}\right)
$$

Note that with the choice of importance density as the prior, the weights were obtained as,

$$
\mathrm{w}_{k}^{i} \propto w_{k-1}^{i} p\left(y_{k}^{d} \mid \theta_{k}^{d, i}, \gamma_{k}^{d, i}\right)
$$

- To avoid the degeneracy problem, a new set of particles is resampled (with replacement) from the approximation of posterior distribution $p\left(\theta_{k}^{d}, \gamma_{k}^{d} \mid y_{0: k}^{d}\right)$ constructed on weighted samples previously drawn, such that weights are reset equally to $\mathrm{w}_{k}^{i}=1 / N$. The objective behind resampling is the elimination of particles with small weights and focus on particles with large weights, for estimation. In this work, systematic resampling scheme is preferred as it is easy to implement and takes $O(N)$ time and the algorithm can be referred in [29].

- The prediction, update and resample procedures form a single iteration step and are applied at each time step $k$. The algorithm for SIR filter is given in Table II. Details about other variants of sequential importance sampling PFs can be referred in [29]. 


\subsubsection{Random Walk Noise Variance Adaptation}

Consider the DPP vector $\gamma^{d} \in \mathbb{R}^{N^{d}}$ such that $\forall j \in\left\{1, . . N_{\gamma^{d}}\right\}, \gamma^{d, j} \in \gamma^{d}, \hat{\gamma}^{d, j}$ is the estimated value, and $\gamma^{d, j^{*}} \in \boldsymbol{\gamma}^{d^{*}}, \boldsymbol{\gamma}^{d^{*}} \in \mathbb{R}^{N_{\gamma^{d}}}$ being the respective true value. Also consider the interval vector $\left[\boldsymbol{\gamma}_{1}^{\mathbf{d}^{*}}, \gamma_{\mathrm{u}}^{\mathbf{d}^{*}}\right] \in \mathbb{R}^{N_{\gamma^{d}}}$, consisting of intervals $\left[\gamma_{l}^{d, j^{*}}, \gamma_{u}^{d, j^{*}}\right] \in\left[\gamma_{1}^{\mathbf{d}^{*}}, \boldsymbol{\gamma}_{\mathbf{u}}^{\mathbf{d}^{*}}\right]$, that contain the true value $\gamma^{d, j^{*}} \in\left[\gamma_{l}^{d, j^{*}}, \gamma_{u}^{d, j^{*}}\right]$. Moreover, for every $\gamma^{d, j} \in \boldsymbol{\gamma}^{d}$, consider an associated constant (proportional gain) $P^{d, j}$ such that $P^{d, j} \in \boldsymbol{P}^{d}, \boldsymbol{P}^{d} \in \mathbb{R}^{N^{d}}$.

$\gamma^{d, j}$ is modeled as a random walk process $\gamma_{k}^{d, j}=\gamma_{k-1}^{d, j}+\xi_{k-1}^{d, j}$ where, $\xi_{k-1}^{d, j}$ is sampled from an artificial random zero-mean Gaussian distribution i.e. $\xi_{k-1}^{d, j} \sim \mathcal{N}\left(0, \sigma_{\xi_{k-1}^{d, j}}^{2}\right)$. Here, $\sigma_{\xi_{k-1}^{d, j}}^{2}$ denotes the associated variance $\mathbf{v}_{k-1}^{\xi^{d, j}}$ at time $k-1$ i.e. $\sigma_{\xi_{k-1}^{d, j}}^{2} \equiv \mathbf{v}_{k-1}^{\xi^{d, j}}$ for notational simplicity where, $\forall j \in\left\{1, . . N_{\gamma^{d}}\right\}, \mathbf{v}^{\xi^{d, j}} \in \mathbf{v}^{\xi^{d}}$ and $\mathbf{v}^{\xi^{d}} \in \mathbb{R}^{N} \gamma^{d}$. Moreover, associated with every $\mathbf{v}^{\xi^{d, j *}}$, consider

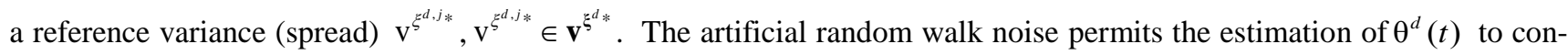
verge to its true value during the estimation process. Selection of the variance of the random walk noise is essentially a tradeoff between values that are big enough to allow the convergence in reasonable amount of time, yet small enough to let the parameter values be tracked smoothly once convergence is reached [18]. One of the efficient ways of ensuring good estimation of $\theta^{d}(t)$ is to reduce the random walk noise variance $\mathrm{v}_{k-1}^{\xi^{d, j}}$, once a suitable convergence is reached. In this regard, performance enhancement has been achieved by the usage of proportional control law type variance adaptation method; it is proposed, demonstrated and implemented in [20]. Therein,

- Variance (spread) is quantified by the statistically robust metric Relative Median Absolute Deviation (RMAD) obtained as,

$$
R M A D(X)=\frac{\operatorname{Median}_{i}\left(\left|X_{i}-\operatorname{Median}_{j}\left(\mathrm{X}_{j}\right)\right|\right)}{\operatorname{Median}_{j}\left(\mathrm{X}_{j}\right)}
$$

where, $X_{i}$ is an element for a data set $X$.

- The variance is adapted in a proportional control law way where the normalized error between the current $\operatorname{RMAD} \mathbf{v}_{k}^{\xi^{d, j}}$ (e.g. $\left.80 \%\right)$ and a reference $\mathbf{v}^{\xi^{d, j *}}$ (e.g. 10\%) is multiplied by a proportional gain constant $P^{d, j}$. Current RMAD $\mathbf{v}_{k}^{\xi^{d, j}}$ is then increased or decreased by that amount. Thereafter, current random walk noise $\xi_{k}^{d, j}$ is sampled from a zero mean Gaussian distribution with the modified variance $\mathrm{v}_{k}^{\xi^{d, j}}$.

However, there-in, the adaptation that progresses in arbitrarily decided multiple stages, requires a proper tuning of reference value $\mathbf{v}^{\xi^{d, j *}}$ and proportional gain constant $P^{d, j}$, for each stage. Such a procedure can be a tedious task especially in presence of multiple DPP. Although the objective that rests in achieving proper convergence and subsequent smooth tracking is clearly achievable, availability of no guidelines for a proper selection of number of stages and $\mathbf{v}^{\xi^{d, j *}}$, makes the task complicated. In this paper, random walk variance is controlled in similar fashion as in [20], however, with the distinguishing feature that variance adaptation is triggered by $\bar{\gamma}_{k}^{d, j}$ :

$\overline{\hat{\gamma}}_{k}^{d, j}= \begin{cases}\frac{1}{L+1} \sum_{l=0}^{l=L} \operatorname{mean}\left(\hat{\gamma}_{k-l}^{d, j}\right) & \text { if } k \geq L \\ \operatorname{mean}\left(\hat{\gamma}_{k}^{d, j}\right) & \text { if } k<L\end{cases}$ 
with $\theta^{d}$ at time $k$, being the average of the estimation mean $\hat{\gamma}^{d, j}$ in a running window of previous $L$ estimates. Fig. 2 shows the schematic of the proposed algorithm. The fact that degradation model of $\theta^{d}(t)$ is known, leads to an approximate knowledge of the true value of $\gamma^{d, j^{*}}$. The adaptation of $\xi^{d, j}$ is triggered when $\overline{\hat{\gamma}}_{k}^{d, j} \in\left[\gamma_{l}^{d, j^{*}}, \gamma_{u}^{d, j^{*}}\right]$. The interval $\left[\gamma_{l}^{d, j^{*}}, \gamma_{u}^{d, j^{*}}\right]$ can be decided based upon the approximate knowledge of $\gamma^{d, j^{*}}$, obtained from the DM. The main objective rests in letting the variance be regulated in an automatic way.

The corresponding pseudo-algorithm of the variance adaptation scheme followed in this paper is given in Table III.

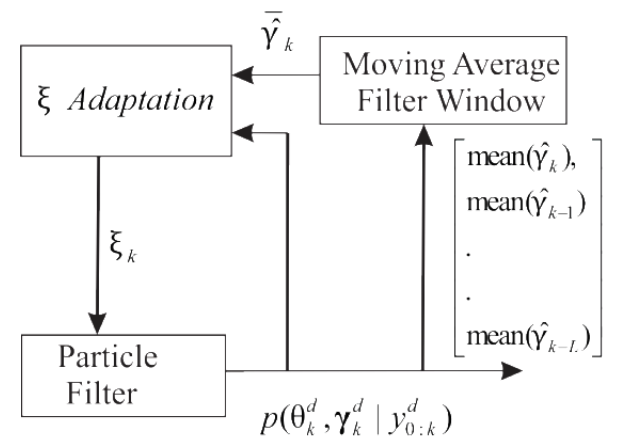

Fig. 2. Schematic of variance control scheme

\subsection{Remaining Useful Life Prediction}

The critical/failure value of $\theta^{d}(t)$ is $\theta_{\text {fail }}^{d}$; it is specified beforehand. The corresponding RUL prediction at time $k$ is $R U L_{k}^{\theta^{d}}$; it is framed as generation of $l^{d}$-step ahead long term prediction $p\left(\theta_{k+l^{d}}^{d}, \gamma_{k+l^{d}}^{d} \mid y_{0: k}^{d}\right)$ based upon the current joint stateparameter estimate $p\left(\theta_{k}^{d}, \gamma_{k}^{d} \mid y_{0: k}^{d}\right)$. The latter is obtained with $l^{d}=1, \ldots T^{d}-k$, where $T^{d}$ is the time horizon of interest i.e. time until $\theta_{k+l^{d}}^{d} \geq \theta_{\text {fail }}^{d}$. The $l^{d}$-step ahead state distribution is computed by propagating each of the particles $\left\{\left(\theta_{k}^{d, i}, \boldsymbol{\gamma}_{k}^{d, i}\right), \mathrm{w}_{k}^{i}\right\}_{i=1}^{N}, l^{d, i}$ steps ahead until $\theta_{k+l^{d i}}^{d, i} \geq \theta_{\text {fail }}^{d}[9,18-20]$ as:

$p\left(\theta_{k+l^{d}}^{d}, \gamma_{k+l^{d}}^{d} \mid y_{0: k}^{d}\right) \approx \sum_{i=1}^{N} w_{k}^{i} \cdot \delta_{\left(\theta_{k+l^{d, i}}^{d, i}, \gamma_{k+l^{d, i}}^{d, i}\right)}\left(d \theta_{k+l^{d}}^{d} d \gamma_{k+l^{d}}^{d}\right)$

where for the $i^{\text {th }}$ particle, the corresponding weight during the $l^{d, i}$-step propagation is kept equal to weight $\mathrm{w}_{k}^{i}$. For $i^{\text {th }}$ particle, $R U L_{k}^{\theta^{d, i}}=k+l^{d, i}-k=l^{d, i}$; the corresponding $R U L_{k}^{\theta^{d}}$ is obtained as:

$p\left(R U L_{k}^{\theta^{d}} \mid y_{0: k}^{d}\right) \approx \sum_{i=1}^{N} \mathrm{w}_{k}^{i} \delta_{\left(R U L_{k}^{\theta^{d}, i}\right)}\left(d R U L_{k}^{\theta^{d}}\right)$

The prediction $R U L_{k}^{\theta^{d}}$ is done in the absence of future observations $y_{k+1: k+l^{d}}^{d}$ which are not available. Pseudo algorithm for RUL prediction is given in Table IV.

\subsection{Health Monitoring Algorithm}

The beginning of degradation is detected by the fault detection module described in Section 2. Subsequently, the joint estimation and RUL prediction is triggered. As the thresholds are sensitive to other uncertain parameters, $\theta_{t=t_{d}}^{d}=\theta_{n}^{d}$ cannot be assured. Thus, the initial value of the state estimate is assumed uniformly distributed as,

$\theta_{t=t_{d}}^{d} \sim U\left(\theta_{n}^{d}-\Delta \theta_{l}, \theta_{n}^{d}+\Delta \theta_{u}\right)$

where $t_{d}$ is the time of degradation commencement. The associated uncertainty interval limits $\left[-\Delta \theta_{l}, \Delta \theta_{u}\right]$ decide the bounds of the uniform distribution as shown in (2). Such an approximation guarantees to include the true initial state of 


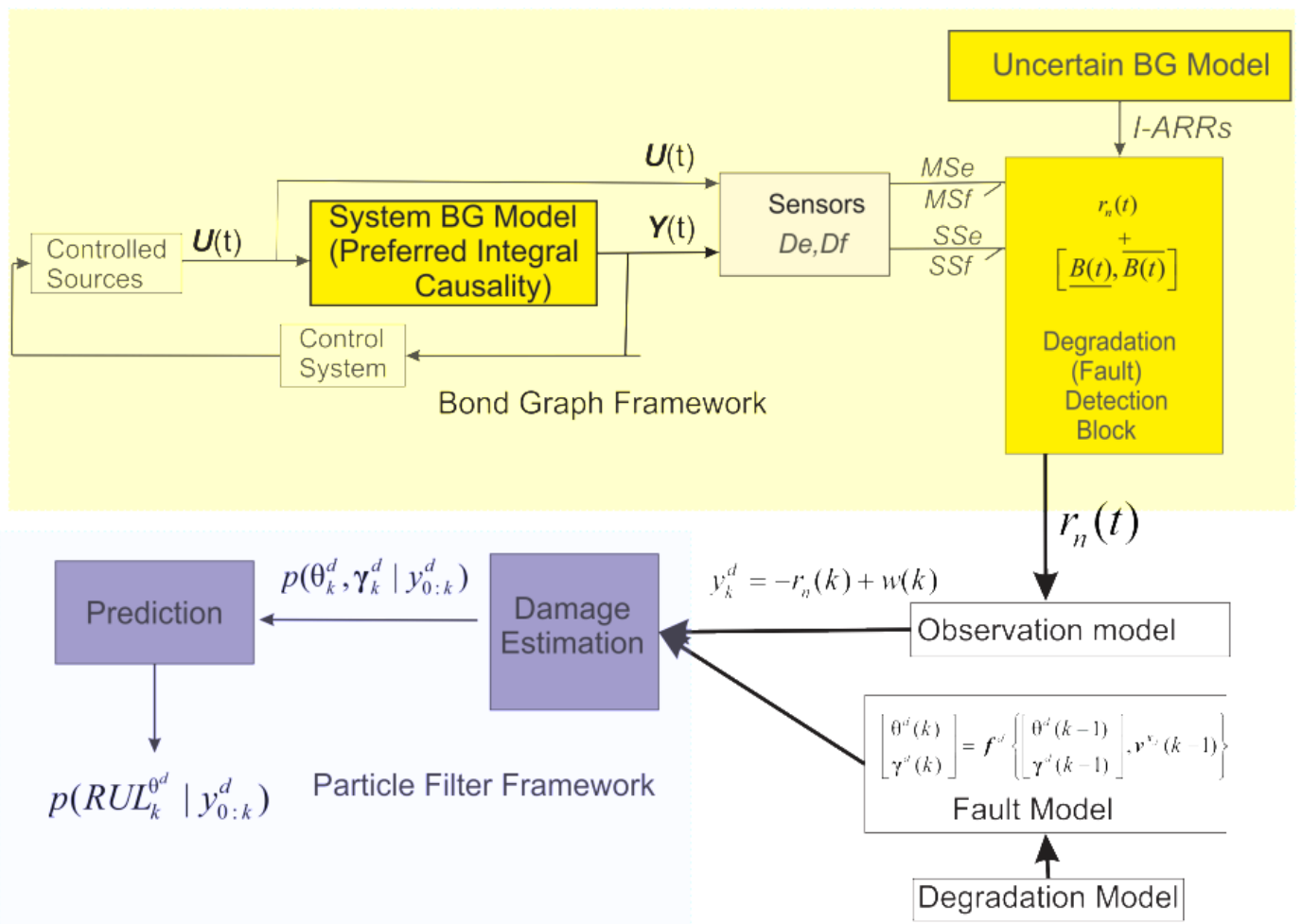

Fig. 3. Schematic description of the Health Monitoring Methodology

\section{Evaluation Metrics}

In this section, various metrics employed to evaluate the performance of estimation, prediction etc. are briefly discussed. [74] can be referred for details and [18, 20] for implementation of the same.

\section{5.1. Estimation performance}

415 The estimation performance is evaluated using two metrics that quantify the accuracy and spread.

416 Root mean square error (RMSE): This metric expresses the relative estimation accuracy as:

$$
\operatorname{RMSE}_{X}=\sqrt{\operatorname{Mean}_{k}\left[\left(\frac{X-X^{*}}{X^{*}}\right)^{2}\right]}
$$

Here, $X \in\left\{\theta^{d}, \gamma^{d}\right\}$ and the corresponding true values are denoted by. Mean ${ }_{k}$ denotes mean over all the values of $k$.

Relative median absolute deviation (RMAD): As detailed in Section 4.1.1, RMAD expresses the spread of estimation relative to median as a percentage. It is averaged over multiple values of $k$ to obtain,

$$
\overline{\operatorname{RMAD}}_{\gamma^{d}}=\operatorname{Mean}_{k}\left(\mathrm{RMAD}_{\gamma^{d}, k}\right)
$$

where $\operatorname{RMAD}_{\gamma^{d}, k}$ is the RMAD of $\gamma^{d}$ at time $k$.

\subsection{Prediction performance}

For a particular prediction time point $k_{p}$, the prediction accuracy is evaluated by Relative Accuracy (RA) metric as, 


$$
\mathrm{RA}_{\theta^{d}, k_{p}}=\left(1-\frac{\left|R U L_{\theta^{d}, k_{p}}^{*}-\operatorname{Mean}\left(R U L_{\theta^{d}, k_{p}}\right)\right|}{R U L_{\theta^{d}, k_{p}}^{*}}\right)
$$

where $R U L_{\theta^{d}, k_{p}}^{*}$ denotes the true $R U L$ at time $k_{p}$, with respect to $\theta^{d}$. The overall accuracy is determined by averaging $\mathrm{RA}_{\theta^{d}, k_{p}}$ over all the prediction points. The latter is denoted as $\overline{\mathrm{RA}}_{\theta^{d}}$; it is determined as shown in (43). The associated spread at $k_{p}$ is denoted as $\mathrm{RMAD}_{\mathrm{RUL} \mathrm{L}^{d}}$. The overall spread is determined by finding the corresponding mean which is denoted as $\overline{\operatorname{RMAD}}_{R U L_{0^{d}}}$

$\overline{\mathrm{RA}}_{\theta^{d}}=\operatorname{Mean}_{k_{p}}\left(\mathrm{RA}_{\theta^{d}, k_{p}}\right)$

\subsection{Prognostics Performance}

$\alpha-\lambda$ metric[74] is employed to assess the prognostic performance. Here, $\alpha \in[0,1]$ defines the bounds of true $R U L$ as $(1 \pm \alpha) R U L_{\theta^{d}, k_{p}}^{*}$ and $\lambda \in[0,1]$ denotes the fraction of time between the initial prediction time point and the true EOL. The third parameter $\beta \in[0,1]$ signifies the desired (pre-fixed) fraction of the RUL prediction probability mass percentage that must fall between the cones of accuracy determined by $\alpha$, for the respective RUL prediction to be acceptable. In this paper, for all $\lambda$ (all $k$ ), $\beta=0.5$ which translates to the requirement of $50 \%$ of probability mass distribution of $R U L_{\theta^{d}, k_{p}}$ falling within $\left[(1-\alpha) R U L_{\theta^{d}, k_{p}}^{*},(1+\alpha) R U L_{\theta^{d}, k_{p}}^{*}\right]$ for the prediction at $k_{p}$, to be acceptable.

\section{Case Study on Mechatronic System}

The method presented in this paper is applied on a mechatronic Torsion Bar 1.0 system shown in Fig.4. [83, 84]; it is integrated with 20SIM, a BG dedicated software [85]. Real time implementation is achieved through 20 SIM 4C 2.1, a prototyping environment that enables C-code implementation in real time on ARM-9 processor based torsion bar system[86]. The interval computations, estimation, variance control and prediction algorithms are written in Matlab Function Block in Simulink. The embedded code is generated through Simulink Coder in Matlab2013a ${ }^{\circledR}$. INTLAB[81, 87] is used to implement interval calculations during simulation. For real time $\mathrm{C}$-code generation, relevant/required functionalities are borrowed from INTLAB.

\subsection{Nominal System}

The schematic model of the mechatronic system (detailed in [84]) is shown in Fig. 5. It consists of the Maxon® servo motor that provides the controlled actuation (rotation) to the disks; it is equipped with voltage amplifier $A_{m}$, inductance $L a$, resistance $R a$, rotor inertia $J_{m}$. The associated motor friction coefficient is $f_{m}$ and torque constant is $k_{m}$. The high stiffness transmission belt provides the torque transmission with the transmission ratio of $k_{\text {belt }}$, to the motor disk with rotational inertia $J_{M d}$.The motor disk is connected to load disk with rotational inertia $J_{L d}$, through a flexible shaft that constitutes the drive train. The shaft is modeled as spring-damper element having damping coefficient $b_{s}$ and spring constant as $k_{s}$ .The friction in the bearings of the motor disk and load disk is modeled as viscous friction with damping parameters as $b_{M d}$ and $b_{L d}$, respectively. Friction arising due to belt action is lumped with the viscous friction coefficient at motor disk in $b_{M d}$. The setup is equipped with motor encoder and load encoder that measure, respectively, the angular position of motor shaft and load disk (2000 pulses per revolution). Angular position of the motor disk is obtained by dividing the motor encoder counts by belt ratio. 
The BG model of the nominal system in integral causality[83] is given in Fig. 6. The control input from PI controller (controlled variable: motor speed $\omega_{m}$ ) modulates the input voltage $M S e: U_{P I}$. The measured angular velocities (obtained from angular position measurements) of motor shaft and load disk are represented as $D f: \omega_{M}$ and $D f: \omega_{L d}$ respectively. Belt is considered of high stiffness and the rigidity is not considered in the model. Also, the frictional loss due to the action of belt is lumped with frictional loss at motor bearing; it is modeled as a resistor element $R: b_{M d}$. GY element models the conversion of electrical current to electrical torque in the DC motor with corresponding coefficient of gyration being $m_{G Y}=k_{m} . T F$ element models the transmission of velocity through the belt from motor shaft to the motor disk. The corresponding coefficient of transformation $m_{T Y}=1 / k_{b e l t}$ where $k_{\text {belt }}$ is the ratio between number of teeth on motor disk to

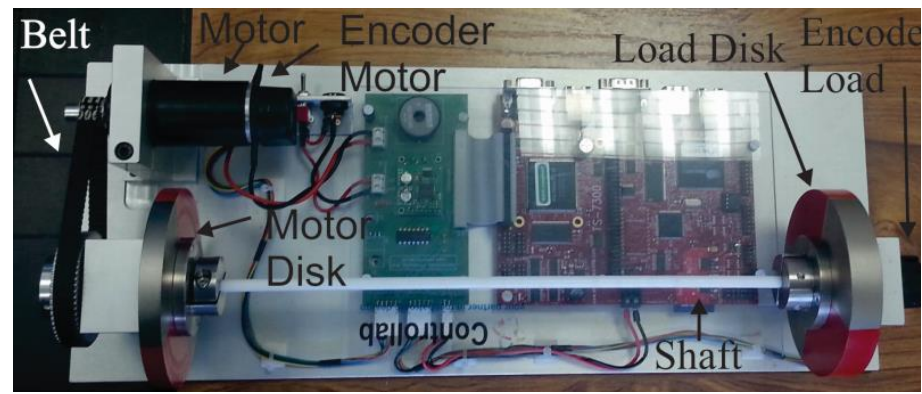

Fig. 4. (a). Mechatronic Torsion Bar 1.0 system

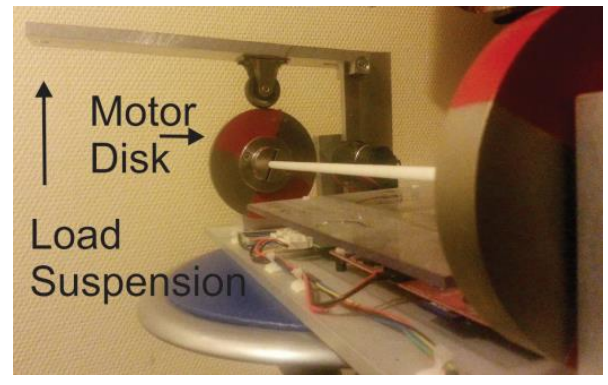

Fig. 4. (b). Fabricated Mechanical Lever type arrangement for Load (Mass) Suspension Belt Friction +
Moto Disk Bearing Friction Shaft Bearing Friction

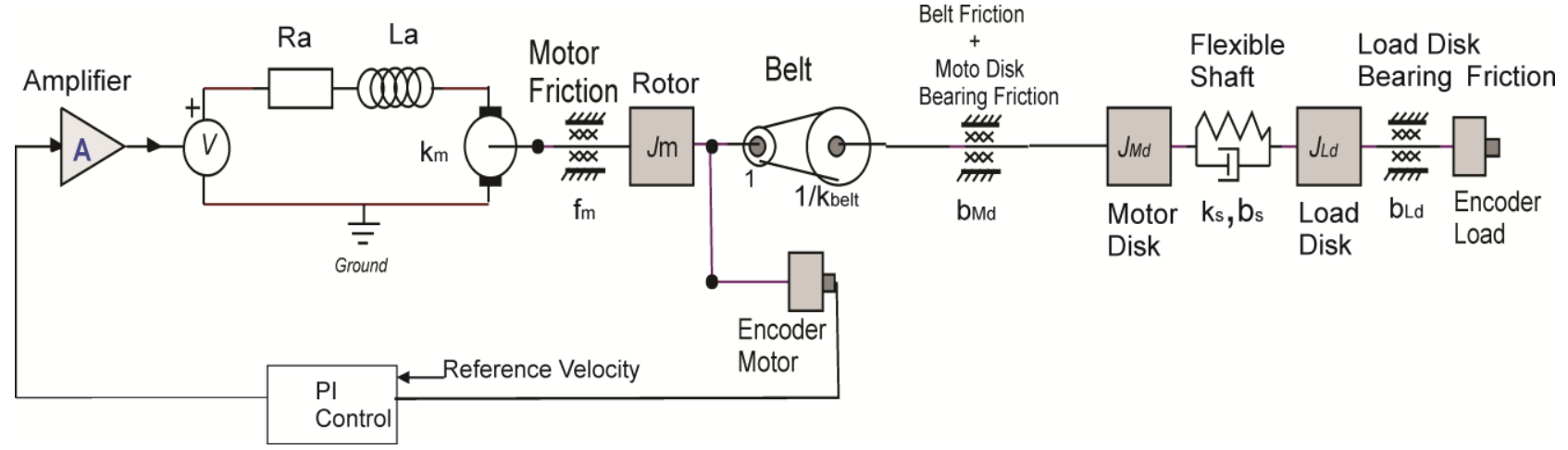

Fig. 5. Schematic Model Of The Mechatronic System

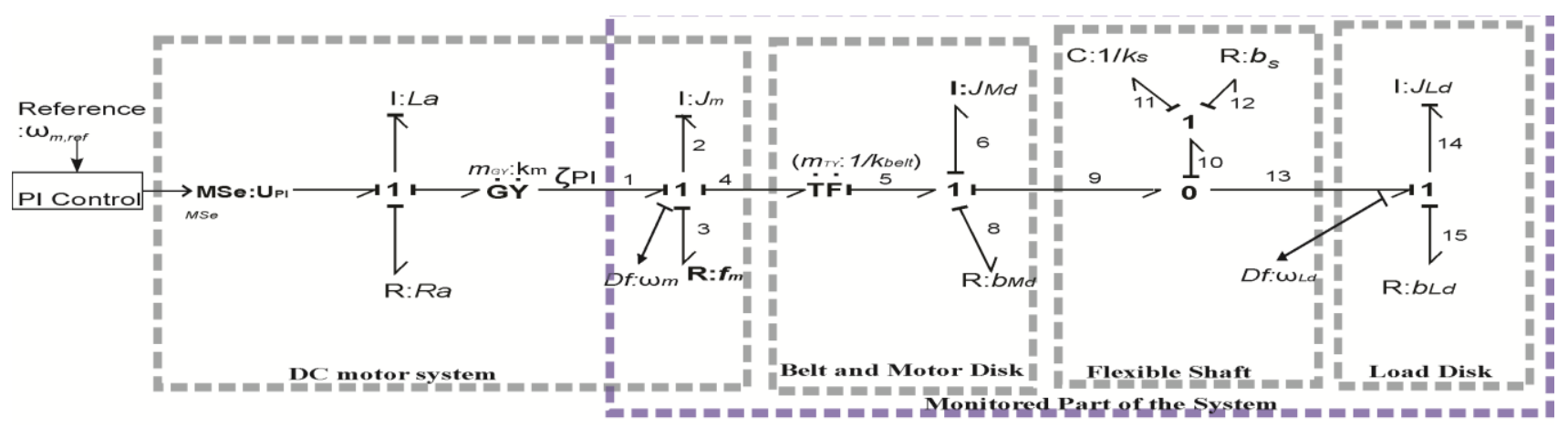

Fig. 6. Bond Graph Model ( Preferred Integral Causality) of The Nominal System 


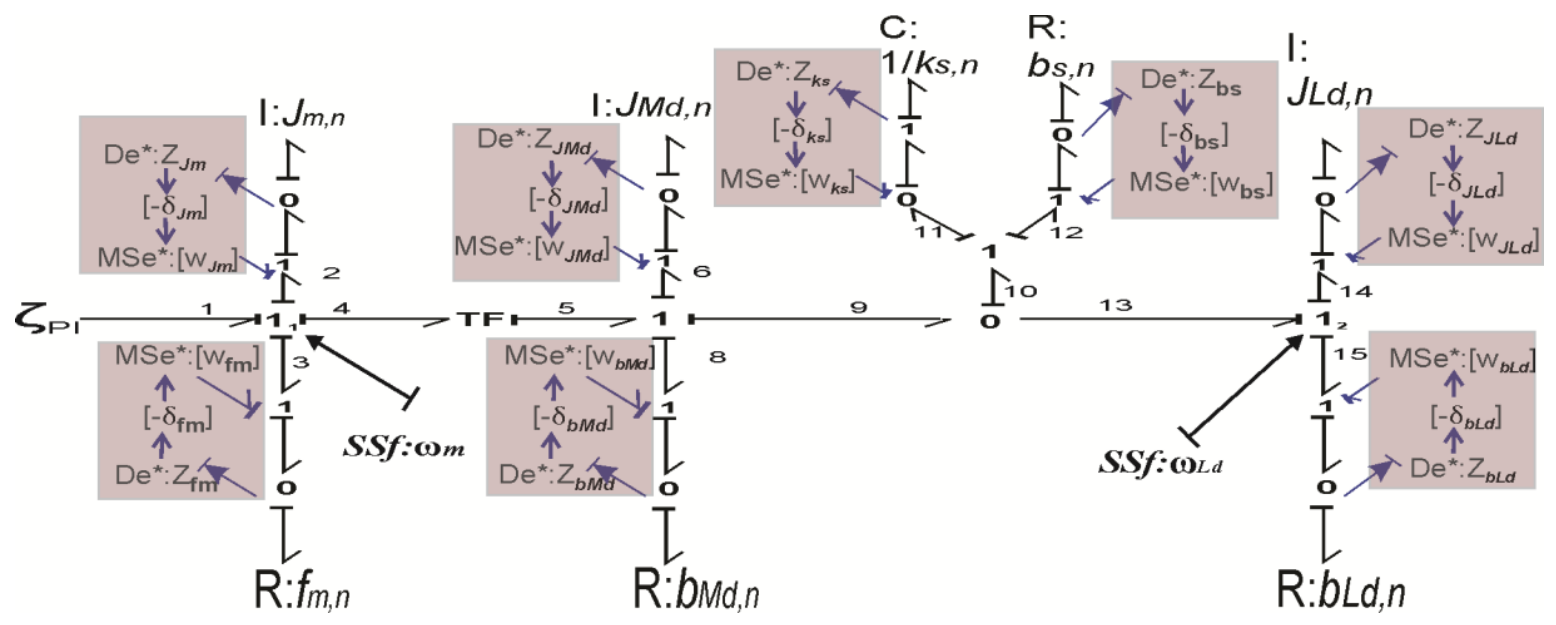

Fig. 7. Bond Graph Model Of Monitorable Part In Preferred Derivative Causality With Parametric Uncertainties As Intervals

Only the monitorable part (marked in Fig. 6) is used for analysis. It must be noted that the system is operating in feedback closed loop (Proportional-Integral (PI) control) regime. Analysis or development of the control strategy is not described, as the main interest of the paper does not lie in the same.

\subsection{Uncertain BG and System Validation}

The uncertain BG of the monitorable part in preferred derivative causality is shown in Fig. 7. The parametric uncertainties are modeled and represented in interval form. The global system is considered uncertain with uncertain parameter

$\operatorname{vector}[\underline{\boldsymbol{\theta}}, \overline{\boldsymbol{\theta}}]:[\underline{\boldsymbol{\theta}}, \overline{\boldsymbol{\theta}}]=\left[[\underline{J m}, \overline{J m}],\left[\underline{f_{m}}, \overline{f_{m}}\right],\left[\underline{J_{M d}}, \overline{J_{M d}}\right],\left[\underline{b_{M d}}, \overline{b_{M d}}\right],\left[\underline{J_{L d}}, \overline{J_{L d}}\right],\left[\underline{b_{L d}}, \overline{b_{L d}}\right],\left[\underline{k_{s}}, \overline{k_{s}}\right],\left[\underline{b}_{s}, \overline{b_{s}}\right]\right]^{T}$

The monitorable part has input in form of the controlled electrical torque input generated by the DC motor. Both the sensors $(D f)$ are dualized to corresponding source of flows as $Y(t)=\left[S S f_{1}: \omega_{m}, S S f_{2}: \omega_{L d}\right]^{T}$. C element remains in integral causality with the initial condition given by the flow at respective $\mathbf{0}$-junction, provided by encoder readings as $f_{10}=f_{9}-f_{13}=\left(\omega_{m} / k_{\text {belt }}\right)-\omega_{L d}$.

\subsubsection{Interval valued ARRs and Robust Thresholds}

From the steps described in Section 2, I-ARR can be generated from the detectable junction $\mathbf{1}_{\mathbf{1}}$ of Fig. 7 as:

$$
\begin{aligned}
& {[\underline{R}, \bar{R}]_{1}=\tau_{P I}-\left(J_{m, n} \dot{\omega}_{m}+\left[\underline{\delta_{J_{m}}}, \overline{\delta_{J_{m}}}\right] J_{m, n} \dot{\omega}_{m}\right)-\left(f_{m, n} \omega_{m}+\left[\underline{\delta_{f_{m}}}, \overline{\delta_{f_{m}}}\right] f_{m, n} . \omega_{m}\right)} \\
& -\left(1 / k_{\text {belt }}\right)\left(\begin{array}{l}
\left(J_{M d, n}\left(\dot{\omega}_{m} / k_{b e l t}\right)+\left[\underline{\delta_{J_{M d}}}, \overline{\delta_{J_{M d}}}\right] J_{M d, n}\left(\dot{\omega}_{m} / k_{b e l t}\right)\right)+\left(b_{M d, n}\left(\omega_{m} / k_{b e l t}\right)+\left[\underline{\delta_{b_{M d},}} \overline{\delta_{b_{M d,}}}\right] b_{M d, n} \cdot\left(\omega_{m} / k_{b e l t}\right)\right) \\
+\left(k_{s, n} \int\left(\frac{\omega_{m}}{k_{b e l t}}-\omega_{L d}\right) d t+\left[\underline{\delta_{k_{s}}}, \overline{\delta_{k_{s}}}\right] k_{s, n} \int\left(\frac{\omega_{m}}{k_{b e l t}}-\omega_{L d}^{\prime}\right) d t+b_{s, n}\left(\frac{\omega_{m}}{k_{b e l t}}-\omega_{L d}\right)+\left[\underline{\delta_{b_{s}}}, \overline{\delta_{b_{s}}}\right] b_{s, n}\left(\frac{\omega_{m}}{k_{b e l t}}-\omega_{L d}\right)\right)
\end{array}\right)
\end{aligned}
$$

Electrical torque $M S e: \tau_{P I}$ is the PI controlled input to the monitorable part of the system; it is given as,

$M S e: \tau_{P I}=k_{m} \cdot i_{m}=k_{m} \cdot \frac{\left(U_{P I}-k_{m} \cdot \omega_{m}\right)}{R a}\left(1-e^{-(R a / L a) \times t}\right)$

where $U_{P I}$ is the PI controlled voltage input and $i_{m}$ is the motor stator current. The nominal part $r_{1, n}(t)$ is formed by collecting point valued nominal parameters as coefficients of known (measured) variables. The I-ARR is expressed as,

$[\underline{R}, \bar{R}]_{1}=r_{1, n}(t)+[\underline{B(t)}, \overline{B(t)}]_{1}$

where, 


$$
[\underline{B(t)}, \overline{B(t)}]_{1}=-\left(\left[\underline{\delta_{J_{m}}}, \overline{\delta_{J_{m}}}\right] J_{m, n} \dot{\omega}_{m}\right)-\left(\left[\underline{\delta_{f_{m}}}, \overline{\delta_{f_{m}}}\right] f_{m, n} . \omega_{m}\right)-\frac{1}{k_{b e l t}}\left(\begin{array}{l}
{\left[\underline{\delta_{J_{M d}}}, \overline{\delta_{J_{M d}}}\right] J_{M d, n} \frac{\dot{\omega}_{m}}{k_{b e l t}}+\left[\underline{\delta_{b_{M d,}}}, \overline{\delta_{b_{M d},}}\right] b_{M d, n} \frac{\omega_{m}}{k_{b e l t}}} \\
\left.\left.+\underline{\delta_{k_{s}}}, \overline{\delta_{k_{s}}}\right] k_{s, n} \int\left(\frac{\omega_{m}}{k_{b e l t}}-\omega_{L d}\right) d t+\left[\underline{\delta_{b_{s}}}, \overline{\delta_{b_{s}}}\right] b_{s, n}\left(\frac{\omega_{m}}{k_{b e l t}}-\omega_{L d}\right)\right)
\end{array}\right)
$$

Only one I-ARR has been derived here at $\mathbf{1}_{\mathbf{1}}$; it serves the purpose of approach-demonstration. Similarly, another independent I-ARR may be derived from $\mathbf{1}_{2}$ junction.

\subsection{Study by Simulation}

\subsubsection{Nominal conditions}

The nominal parameter values and respective multiplicative interval uncertainty is tabulated in Table VI. Fig. 8 shows the nominal outputs where motor velocity $\omega_{m}$ is PI controlled with the reference $\omega_{m, r e f}=112.5 \mathrm{rad} / \mathrm{s}$. Then, the motor disk velocity $\omega_{M d}$ is regulated to $\omega_{M d, r e f}=\omega_{m, \text { ref }} / k_{\text {belt }}=30 \mathrm{rad} / \mathrm{s}$. Noise is added to sensor outputs. It corrupts the residual and is approximated as $w^{d}(t) \sim \mathcal{N}\left(0, \sigma_{w^{d}}^{2}\right) ; \sigma_{w^{d}}=0.01 \mathrm{~V}$. Negative value of residual $-r_{1, n}^{d}(t)$ is contained within the interval threshold bounds determined in (49).
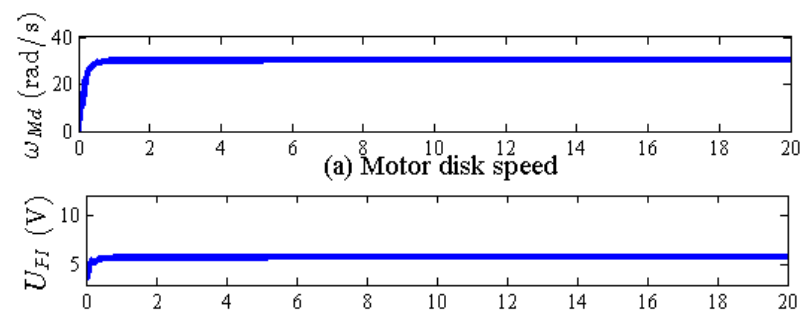

(b) Input voltage (PI-controlled)

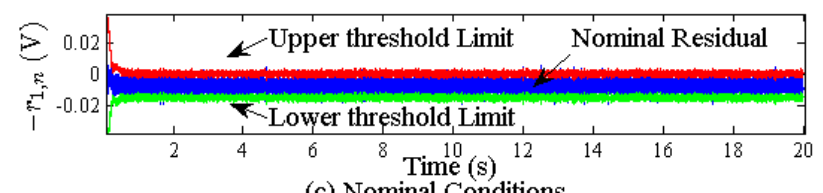

(c) Nominal Conditions

Fig. 8 Nominal Conditions (a) Motor disk speed (b) Input voltage (c) Nominal residual and Interval valued thresholds
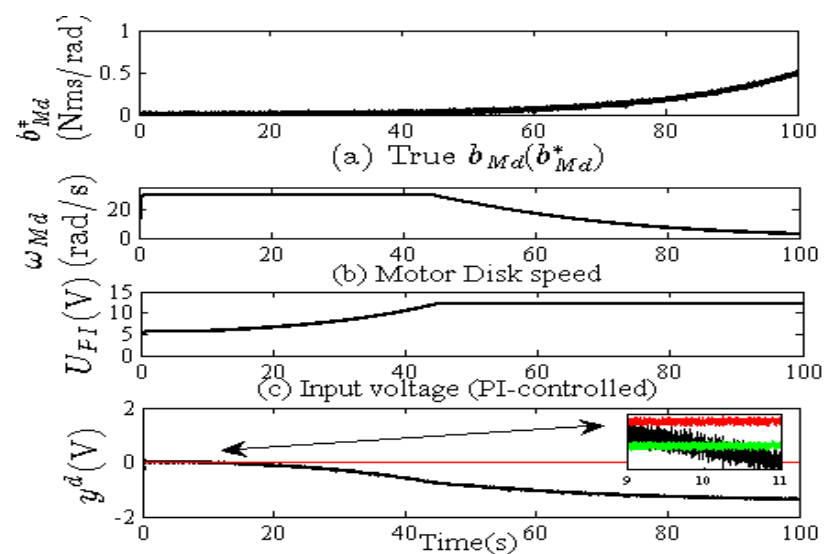

(d) Nominal part of I-ARR under degradation

Fig. 9. Simulation of Degradation (a) Injected Degradation (b) Motor Disk Speed (c) Input Voltage to the System (d) Nominal Residual $r_{1, n}(t)$

\subsubsection{Generation of Parametric Degradation}

Degradation of motor disk bearing friction parameter $b_{M d}$, is simulated by considering the degradation model exponential in nature as,

$$
b_{M d}(t)=\left\{\begin{array}{l}
g_{1}\left(b_{M d}, \gamma_{1}\right)+v_{b_{M d}} \\
b_{M d, n} e^{\gamma_{1}(t)}+v_{b_{M d}}
\end{array}\right.
$$

where, $g_{1}$ is the DM, $\theta^{d}(t)=b_{M d}$ is the state variable and DPP vector $\gamma^{d}=\left\{\gamma^{d}\right\}=\gamma_{1}$ and $v_{b_{M d}} \sim \mathcal{N}\left(0, \sigma_{v_{b_{M d}}}^{2}\right)$ is the process noise. Fig. 9 shows the corresponding outputs. The fault is detected at $t_{d}=10 \mathrm{~s}$ when residual crosses the interval thresholds. Note that $\omega_{M d}$ is controlled at $30 \mathrm{rad} / \mathrm{s}$ until $t=44.2 \mathrm{~s}$ while the PI controller is effective. Thereafter, as the saturation value of actuator (motor) input voltage $(12 \mathrm{~V})$ is reached, the speed $\omega_{M d}$ starts to decrease and reaches $\omega_{M d \text {,stop }}=3 \mathrm{rad} / \mathrm{s}$ at 
$t=100$ s. The latter is the time point at which system is considered to have obtained the failure state. The residual is sensitive to the input torque and hence, the input voltage captures the degradation evolution throughout the system's lifetime.

\subsubsection{Fault model}

State measurement is obtained from the observation equation which is developed using the Nominal Part of I-ARR $[\underline{R}, \bar{R}]_{1}, r_{1, n}(t)($ cf. $(25)$ and (27)) as,

$$
\begin{aligned}
& 0=r_{1, n}(t)+\left(b_{M d}(t)-b_{M d, n}\right) \cdot \frac{\partial\left(r_{1, n}(t)\right)}{\partial\left(b_{M d}\right)} \\
& y^{d}=-r_{1, n}(t)+w^{d}(t)=\left(b_{M d}(t)-b_{M d, n}\right) \frac{-\omega_{m}(t)}{k_{b e l t}^{2}}+w_{1}(t)
\end{aligned}
$$

where $w_{1}(t) \sim \mathcal{N}\left(0, \sigma_{w_{1}}^{2}\right)$ approximates the noise which corrupts $-r_{1, n}(t)$. For estimation, the fault model denoted as tuple $\left(b_{M d}(t), \gamma_{1}, g_{1}\right)$, is formulated as,

$$
b_{M d, k}=b_{M d, k-1} \cdot e^{\gamma_{1, k-1} \Delta t}+v_{b_{M d}, k-1}
$$

$$
\begin{aligned}
& \gamma_{1, k}=\gamma_{1, k-1}+\xi_{1, k-1} \\
& y_{k}^{d}=\left(b_{M d, k}-b_{M d, n}\right) \frac{-\omega_{m, k}}{k_{b e l t}^{2}}+w_{1, k}
\end{aligned}
$$

where, $\xi_{1}(t) \sim \mathcal{N}\left(0, \sigma_{\xi_{1}}^{2}\right)$ is the additive random walk noise. The estimation of state of parameter $b_{M d}(t)$ is triggered at $t_{d}=10 \mathrm{~s}$. Initial estimate $b_{M d, t_{d}=10 s} \sim U(0.045,0.055) \mathrm{N} . \mathrm{m} . \mathrm{s} / \mathrm{rad}$, contains $b_{M d, n}=0.005 \mathrm{~N} . \mathrm{m} . \mathrm{s} / \mathrm{rad}$. The true value of DPP is kept as $\gamma_{1}^{*}=0.05 \mathrm{Nm} / \mathrm{rad}$ so that $\omega_{M d, s t o p}$ is reached at $100 \mathrm{~s}$. Here, $\Delta t=0.1 \mathrm{~s}$ and $N=500$. Simulation is run until $t_{f}=100 \mathrm{~s}$ when $\hat{b}_{M d}$ reaches the failure value $b_{M d, f a l}=0.45$ N.m.s/rad.

\subsubsection{Degradation Estimation}

Estimated $\hat{b}_{M d}$ is shown in Fig. 10. The true state $b_{M d}^{*}$ is estimated accurately with $R M S E_{b_{M d}}=4.21 \%$. In fact, estimation spread decreases as the estimation progresses, indicating the desirable performance. Estimation of $b_{M d}$ largely depends upon quality of estimation achieved with $\gamma_{1}$. Fig. 11 shows the estimation of $\gamma_{1}$ achieved with $\left[\gamma_{1, l}^{*}, \gamma_{1, u}^{*}\right]=[0.03,0.07]$ N.m./rad, proportional gain $P_{1}=0.001, \mathrm{v}^{\xi_{1}^{*}}=10 \%$, initial artificial noise variance $\sigma_{\xi_{1}, k=0}^{2}=0.02^{2}$. The particle filter assumes measurement noise variance equal to 4 times that of residual noise variance $\sigma_{w_{1}}^{2}=0.01^{2}$. The convergence is achieved very quickly but with large initial estimation spread. This is due to the high artificial noise variance set for the desirable quick convergence. As shown in Fig. 11b, the estimation spread is reduced (effective from $\mathrm{t}=20 \mathrm{~s})$ until $\mathrm{v}^{\xi_{1}^{*}}=10 \%$ is achieved at around $t=50 \mathrm{~s}$ and thereafter, $\gamma_{1}^{*}$ is tracked smoothly with controlled spread and $R M S E=3.02 \%$. For comparison, Fig. 11c shows performance with no variance adaptation. Therein, the estimation continues with large spread even after the convergence is achieved.

\subsubsection{RUL prediction}

Using $\alpha=0.1, \beta=0.5$ and for all $\lambda_{k}$, RUL prediction is shown in Fig. 12. The RUL predictions obtained until $t=52 \mathrm{~s}$ are not good and suffer with large variance spread due to the large corresponding spread in $\hat{\gamma}_{1}$ (see Fig. 11a), making them virtually useless. However, after $t=52 \mathrm{~s}$, the RUL distributions are well within accuracy cone (more than $50 \%$ of RUL 
probability mass lies within accuracy cone). Ignoring the initial period of convergence, the overall prediction performance is very good with $\overline{R M A D}_{R U L}=9.8 \%$ and $\overline{R A}=97.15 \%$.

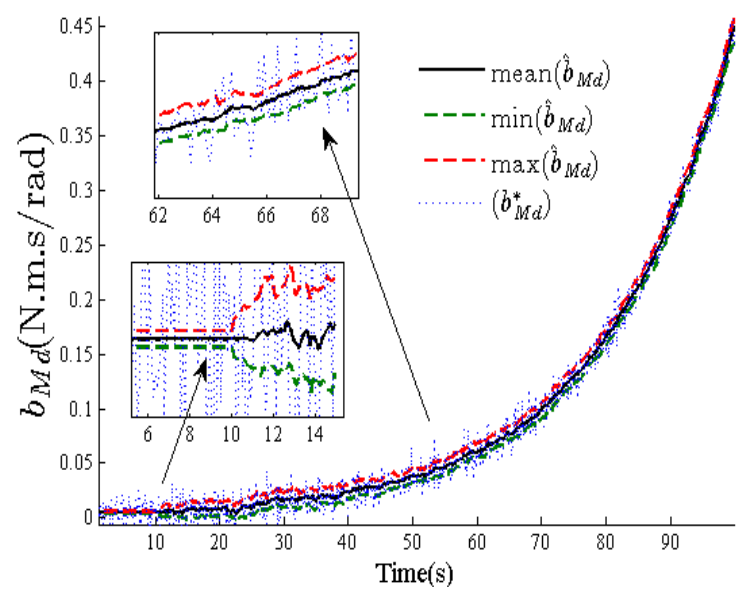

Fig. 10. State estimation of the prognostic candidate system parameter $b_{M d}$
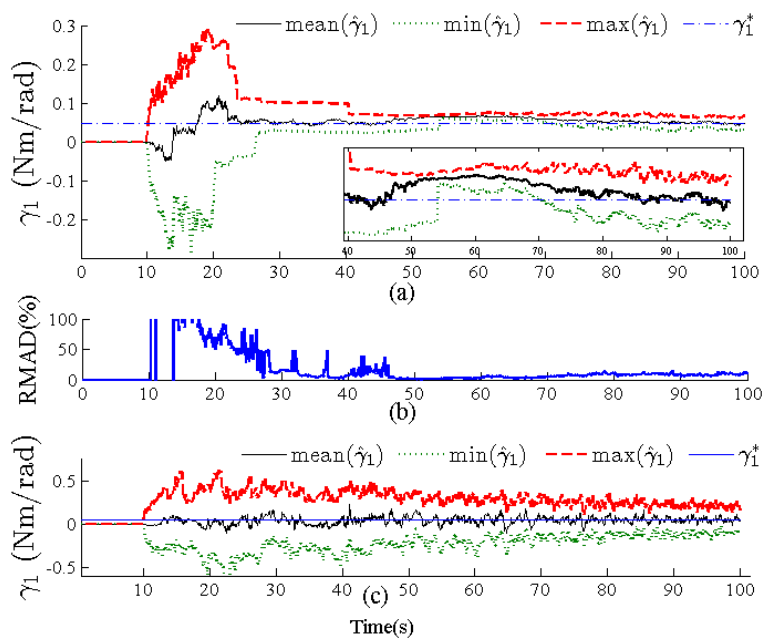

Fig. 11. Estimation performance (a) Estimation of DPP with variance adaptation (b) Estimation spread associated (c) Estimation performance without variance adaptation only for comparison purpose

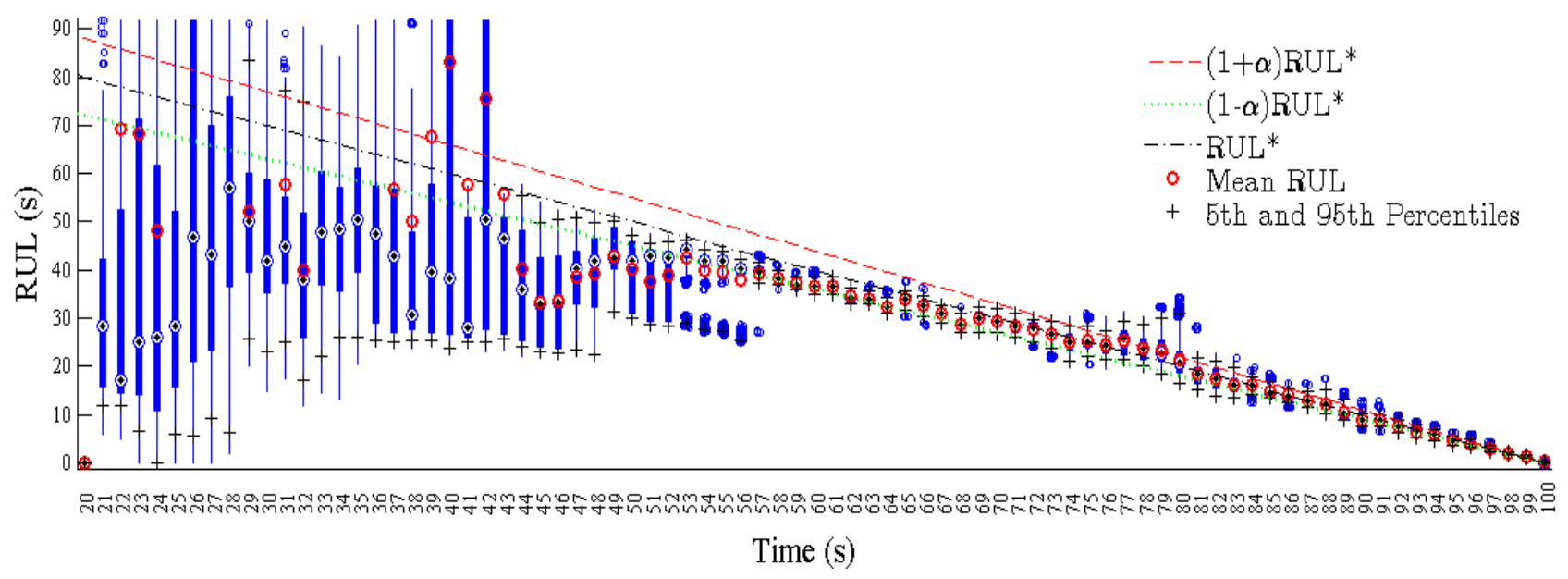

Fig. 12. RUL Prediction performance with respect to estimation in Fig. 10 and Fig. 11. (a).

- Initial variance of the artificial random walk noise, $\sigma_{\xi, k=0}^{2}\left(\right.$ or $\left.v_{k=0}^{\xi_{1}}\right)$ : The initial variance of random walk noise is set according to the magnitude order of DPP $\gamma^{d, j^{*}}$. It is kept high enough so that $\overline{\hat{\gamma}}_{1, k}$ is captured quickly as 
$\overline{\hat{\gamma}}_{1, k} \in\left[\gamma_{1, l}^{*}, \gamma_{1, u}^{*}\right]$. It is the most important factor that determines good tuning of parameters in succession. A very high value of the latter, results in bad estimation performance. Fig. 13a shows estimation with $\sigma_{\xi, k=0}^{2}=0.04^{2}$ (read high) wherein, although, quick convergence of mean $\hat{\gamma}_{1}$ is seen, the estimation continues further with a very wide spread for a long time before it is gradually reduced, owing to variance adaptation scheme. On the contrary, a very low variance will result in very late convergence, if at all. Fig. $13 \mathrm{~b}$ has $\sigma_{\xi, k=0}^{2}=0.001^{2}$ leading to a very late convergence. For tuning of other related parameters in this paper, an initial high value of variance $\sigma_{\xi_{1}, k=0}^{2}$ is chosen.

- Proportional Gain P: Proportional gain determines how fast the estimation spread is reduced to the reference $\mathbf{v}^{\xi^{*}}$ . As observed in Fig. 11a, an appropriate choice of latter was found as $P_{l}=0.001$. It resulted in smooth tracking after convergence was achieved. A high gain value results in quick reduction of estimation spread; however, it is accompanied with continuous shrink and expansion as shown in Fig. $13 \mathrm{c}$ with $P_{I}=0.005$. The latter has also been demonstrated in [20]. Although, a very high gain value may bring down variance spread quickly; however, it may be followed by poor convergence results as shown in Figs. $14 \mathrm{c}$ and d, with $P_{I}=0.01$. On the contrary, a very low $P_{l}$ renders a non-effective variance adaptation as shown in Fig. $13 \mathrm{~d}$ with $P_{l}=0.0001$, adding no significant benefits in RUL prediction.

- Desired RMAD ( $\mathrm{v}^{\xi^{*}}$ ): The pre-fixed $\mathrm{v}^{\xi_{1}^{*}}$ for $\xi_{1}$, determines how much freedom is given to $\gamma_{1}$ after the estimation spread is brought under control. An appropriate choice of $v^{\xi_{1} *}$ gives enough freedom for convergence even after actual variance is well under $\mathrm{v}^{\xi_{1}^{*}}$, as shown in Fig. 11a between $t=50 \mathrm{~s}$ and $t=80 \mathrm{~s}$ with $\mathrm{v}^{\xi_{1}^{*}}=10 \%$. In extreme cases, where $P_{l}$ is chosen of high value (rate of RMAD reduction depends on $P_{1}$ ) and $\mathrm{v}^{\xi_{1}^{*}}$ is set very low, the estimation may remain stagnant near, but not equal to $\gamma_{1}^{*}$. This is shown in Fig. $14 \mathrm{c}$ with $P_{l}=0.01$ (read high) and desired RMAD $v^{\xi_{1}^{*}}=6 \%$ (read very low).

- True DPP interval $\left[\gamma_{1, l}^{*}, \gamma_{1, u}^{*}\right]$ : The main objective of the latter remains in triggering the variance adaptation. As such, if width of $\left[\gamma_{1, l}^{*}, \gamma_{1, u}^{*}\right]$ is kept too tight around $\gamma_{1}^{*}, \overline{\hat{\gamma}}_{1, k}$ may never be captured inside the $\left[\gamma_{1, l}^{*}, \gamma_{1, u}^{*}\right]$ band. This may lead to a very insignificant effect of variance adaptation on the estimation performance. Fig. 14a shows the estimation with $\left[\gamma_{1, l}^{*}, \gamma_{1, u}^{*}\right]=[0.04,0.06] \mathrm{Nm} / \mathrm{rad}$, which can considered "too tight" around $\gamma_{1}^{*}=0.05 \mathrm{Nm} / \mathrm{rad}$. Here, the variance adaption is not effective enough. On the contrary, if the interval width is appropriately set (assuming that initial estimate is outside of it), $\overline{\hat{\gamma}}_{1, k}$ is captured quickly and variance control is triggered early, as shown in Fig. 14b with $\left[\gamma_{1, l}^{*}, \gamma_{1, u}^{*}\right]=[0.01,0.09] \mathrm{Nm} / \mathrm{rad}$. This leads to early reduction in variance. However, a bad choice of $P_{1}$ (read high) and early variance adaptation, may lead to a rapid reduction in spread, followed by stagnation of estimation around $\gamma^{d, j^{*}}$, before converging slowly to the same, as shown in Fig. 14d with $P_{l}=0.005$ and $\left[\gamma_{1, l}^{*}, \gamma_{1, u}^{*}\right]=[0.01,0.09] \mathrm{Nm} / \mathrm{rad}$.

- Residual noise variance (measurement noise) assumed by PF: Noise that corrupts the residual measurements can be non-Gaussian due to presence of derivative terms. Such noises can be dealt by PF effectively without any restrictions. In this work, the explicit distribution of the residual noise is not found. Instead, it is approximated as 
normally distributed Gaussian in nature. The related standard deviation and variance is found out from residual measurements. Moreover, generally, the variance of measurement noise (residual noise here) assumed by PF, is greater than the approximated measurement noise. This is done to counter the sample impoverishment problem which happens while very few particles have significant weights and most of the other particles with nonsignificant weights are abandoned during the resampling process [88]. Higher residual noise variance assumed by $\mathrm{PF}$ allows higher particles being sampled for estimation, thus, reducing the problem of sample degeneracy and consequent impoverishment. As followed in other works [20], in this work too, the residual noise assumed by PF is greater than actual residual noise.

\subsection{Computational complexity}

The time taken per step for estimation and RUL prediction depends on the number of particles used. With $N=500$, on an average, $0.03 \mathrm{~s}$ was consumed per step. Fig. 15 shows the RUL prediction computation time per step for the RUL prediction performance of Fig. 12. In addition to the number of particles $N$, computational time for RUL prediction varies:

- Inversely with the time at which prediction is made: The farther is the time from EOL at which RUL prediction is made, the longer it takes to simulate to EOL. This makes the computational time large.

- Inversely with estimated DPP $\hat{\gamma}:$ At a certain time of prediction, higher is the rate of damage progression, smaller is time taken to simulate to EOL. As seen in Fig. 11a, before $t=50 \mathrm{~s}$, the estimation value of $\hat{\gamma}_{1}$ is lower than true value accompanied with large variance. Therefore, for a specific $N$, the computation time per step before $t=50 \mathrm{~s}$ is higher and with large variations. After $t=50 \mathrm{~s}$, with a nearly uniform $\hat{\gamma}_{1}$ estimation and lesser spread (see Fig. 11a), the computation time follows an almost uniform monotonic decreasing trend (see Fig. 15).

Simulations were run on a $2.49-\mathrm{GHz}$ dual core processor with $8 \mathrm{~GB}$ RAM. With $N=500$, and sample time of $0.1 \mathrm{~s}$ (which translates to 10 computational steps per second); it took on an average 32 minutes to simulate system dynamics, estimation and RUL prediction till 100s. With $N=50$, the same took 110 seconds. This indicates that through employment of lesser number of particles, the RUL predictions could be achieved in real time, for experimental purposes. Moreover, for real experiments run on complied $\mathrm{C}$, the run time reduces drastically by an order of magnitude.

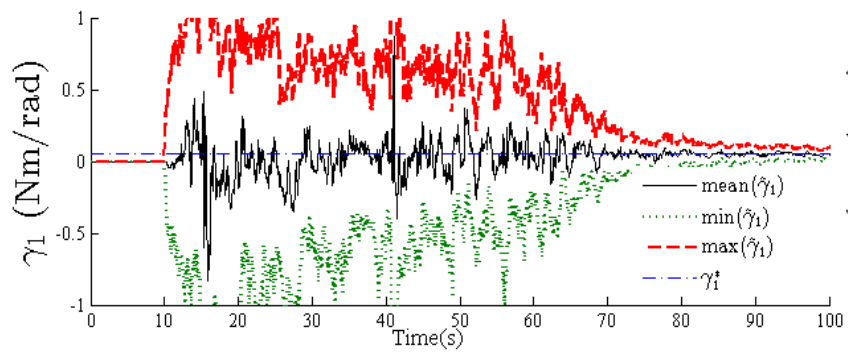

(a) Estimation with large initial random walk variance

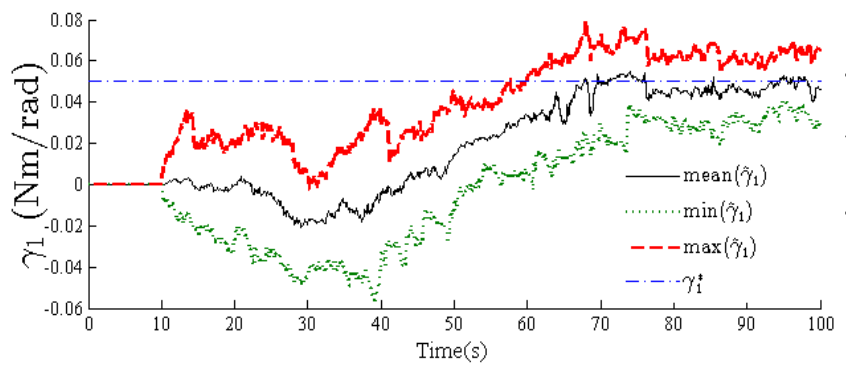

(b) Estimation with small initial random walk variance

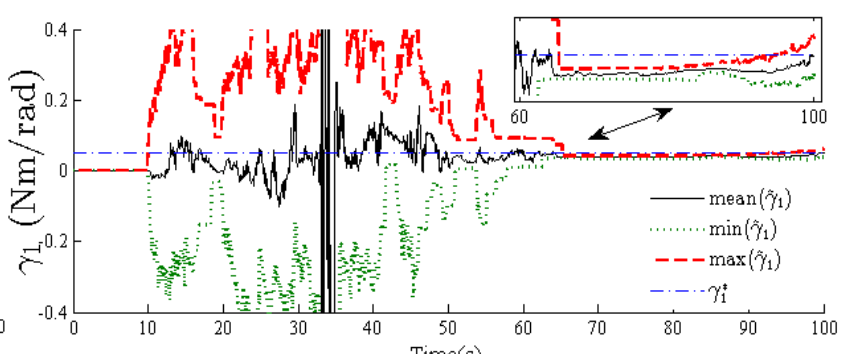

(c) Estimation with high proportional gain in Variance control

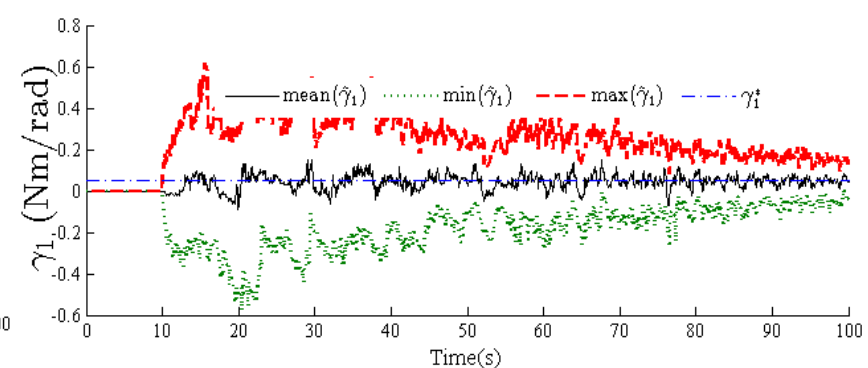

(d) With very low proportional gain in variance control

Fig. 13. Estimation of DPP $\gamma_{1}$ for qualitative analysis (a) Large initial random walk variance $\sigma_{\xi_{1}, k=0}=0.04 \mathrm{Nm} / \mathrm{rad}$ (b) Small initial random walk 
variance $\sigma_{\xi_{1}, k=0}=0.001 \mathrm{Nm} / \mathrm{rad}$ (c) High proportional gain in variance control $P_{l}=0.005$ (d) Very low proportional gain in variance adaptation $P_{l}=0.0001$

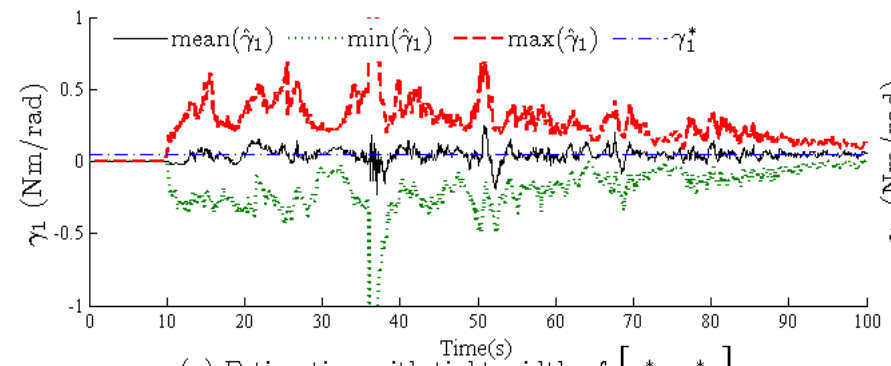

(a) Estimation with tight width of $\left[\gamma_{1, l}^{*}, \gamma_{1, u}^{*}\right]$

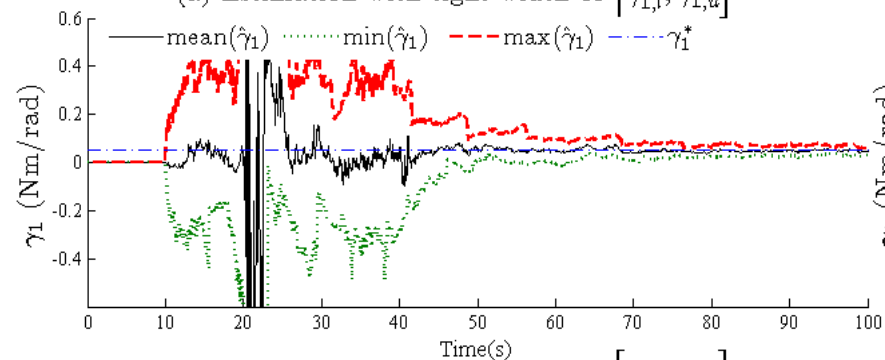

(b) Estimation with wide width of $\left[\gamma_{1, l}^{*}, \gamma_{1, u}^{*}\right]$

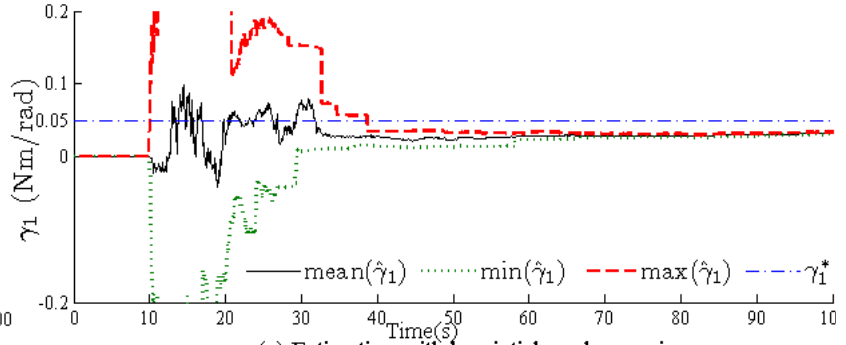

(c) Estimation with low intial random variance

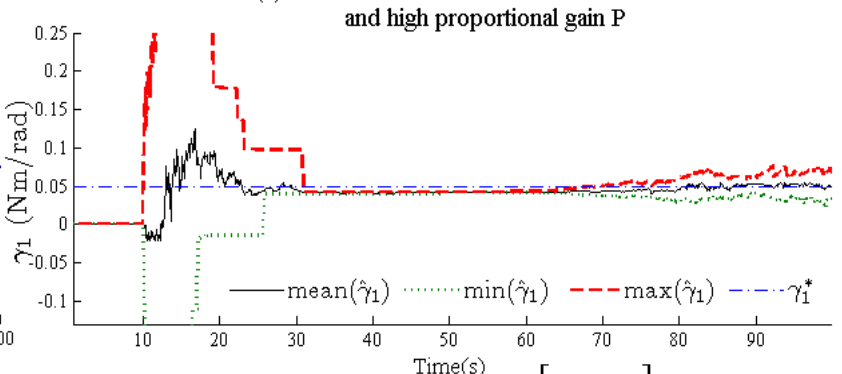

(d) Estimation with large width of $\left[\gamma_{1, i}^{*}, \gamma_{1, u}^{*}\right]$ and high proportional gain $\mathrm{P}$ in variance control

Fig. 14. Estimation of DPP $\gamma_{1}$ for qualitative analysis (a)Tight width $\left[\gamma_{1, l}^{*}, \gamma_{1, u}^{*}\right]=[0.04,0.06] \mathrm{Nm} / \mathrm{rad}$ (b) Wide width with $\left[\gamma_{1, l}^{*}, \gamma_{1, u}^{*}\right]=[0.01,0.09] \mathrm{Nm} / \mathrm{rad}$ (c) High gain value, $P_{l}=0.01$ and very low desired RMAD $\mathrm{v}^{\xi^{*}}=6 \%$ (d) High gain $P_{l}=0.005$ and large width $\left[\gamma_{1, l}^{*}, \gamma_{1, u}^{*}\right]=[0.01,0.09] \mathrm{Nm} / \mathrm{rad}$

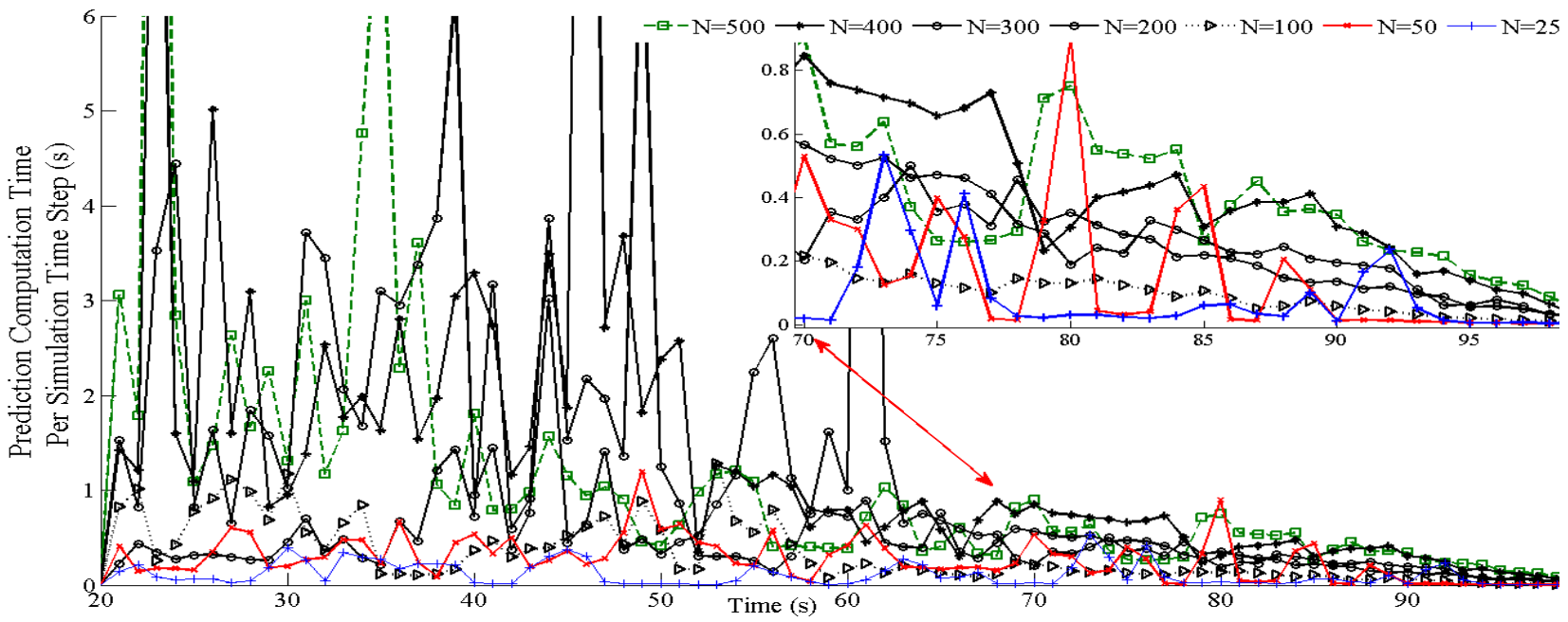

Fig. 15 Prediction computational time for per step for different number of particles 


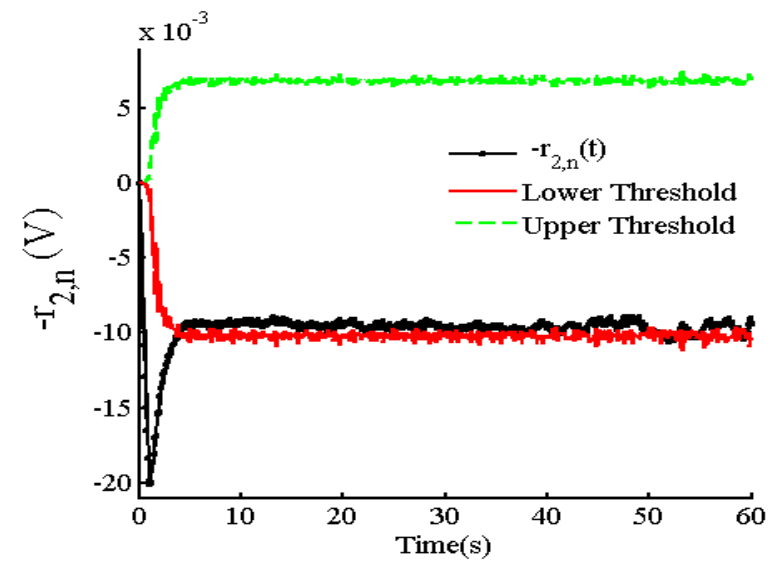

Fig. 16. Nominal residual $-r_{2, n}(t)$ under nominal conditions
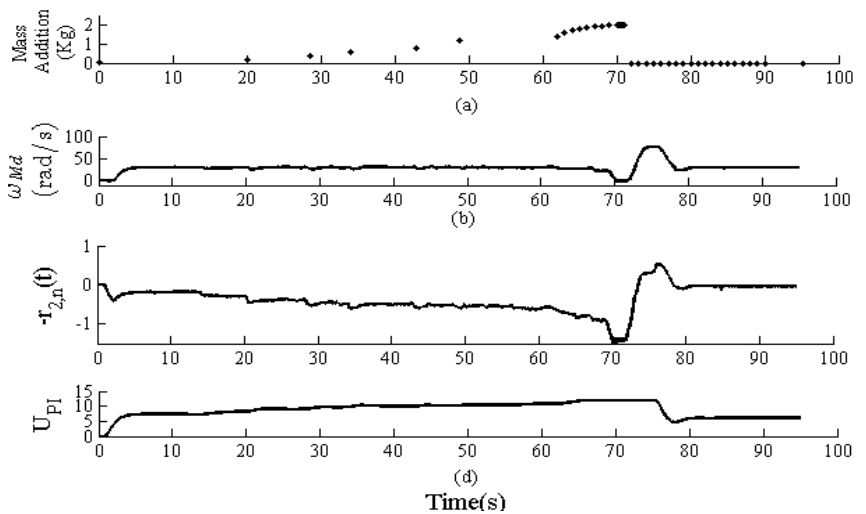

Fig 17. (a) Addition of mass discretely to introduce degradation (b). Motor disk speed (c) Nominal residual $-r_{2, n}(t)$ (d) Input voltage (PI controlled)

\section{Experiments and Results}

For the experiments, a mechanical lever type arrangement is fabricated as shown in Fig. 4b; it introduces frictional torque $\tau_{\text {Mech }}$ over the motor disk by suspension of load in form of sand. The frictional torque manifests due to Coulomb friction existing between the surfaces ( $\mu$ being friction coefficient); it is modulated by the suspended load of mass $M \mathrm{~kg}$ as,

$$
\begin{aligned}
\tau_{\text {Mech }} & =f_{\text {mech }} \cdot r_{M d} \\
f_{\text {mech }} & =\mu M g\left(\omega_{M d} /\left|\omega_{M d}\right|\right)
\end{aligned}
$$

with $r_{M d}$ as the radius of the motor disk. In the BG model, it is incorporated as non-linear resistance element at motor disk as shown in (55); the corresponding characteristic equation is obtained as shown in (56).

$$
\begin{aligned}
& R=b_{M d}+\mu M(t) . r_{M d} g /|\omega| \\
& e_{8}=R\left(f_{8}\right)=b_{M d} \omega_{M d}+\mu M(t) . r_{M d} g \times\left(\omega_{M d} /\left|\omega_{M d}\right|\right)
\end{aligned}
$$

The corresponding I-ARR $[\underline{R}, \bar{R}]_{1}$ in $(45)$ changes to $[\underline{R}, \bar{R}]_{2}$ as,

$r_{2, n}(t)=\tau_{\text {in }}-J_{m, n} \dot{\omega}_{m}-f_{m, n} \omega_{m}-\frac{1}{k_{b e l t}}\left(\begin{array}{l}J_{M d, n} \frac{\dot{\omega}_{m}}{k_{b e l t}}+b_{M d, n} \frac{\omega_{m}}{k_{b e l t}}+\mu_{n} M_{n} g r_{M d} \operatorname{sgn}\left(\omega_{m} / k_{\text {belt }}\right) \\ +k_{s, n} \int\left(\frac{\omega_{m}}{k_{\text {belt }}}-\omega_{L d}\right) d t+b_{s, n}\left(\frac{\omega_{m}}{k_{\text {belt }}}-\omega_{L d}\right)\end{array}\right)$

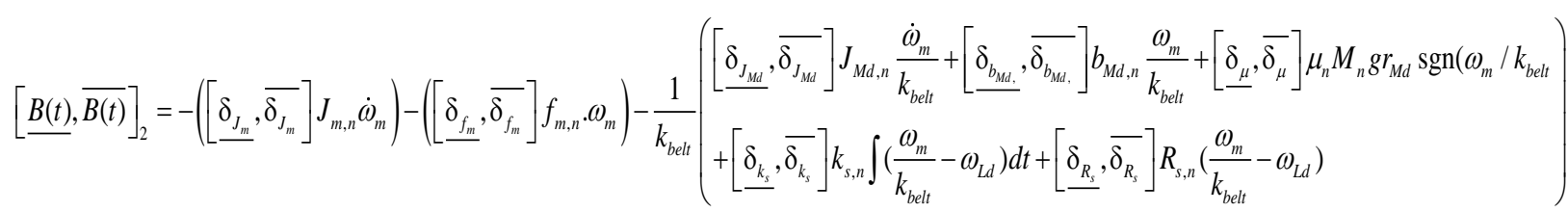

The nominal value of $\mu, \mu_{n}$ is found out from $r_{2, n}(t)$ and (57) by suspending a known load mass. Fig. 16 shows the residual profile under nominal conditions. Fig. 17 shows the effect of adding load (or frictional toque) in a discrete way on the system. $\omega_{M d}$ is controlled at $30 \mathrm{rad} / \mathrm{s}$. Each time load is added, there is PI controller enabled compensation due to which $\omega_{M d}$ settles to the reference velocity. However, $-r_{2, n}(t)$ being sensitive to increase in current (and thus, voltage) decreases and settles to a different value. Saturation value for input voltage is reached around $t=65 \mathrm{~s}$ as the total load suspended is 1.6 $\mathrm{Kg}$. Thereafter $(t>65 \mathrm{~s})$, controller is unable to compensate the change in $\omega_{M d}$. Addition of more sand leads to reduction in motor disk speed; it stops at around $t=70$ s. For safety reasons, the disk is kept at stop condition for few seconds after 
which the load is removed; this brings back the controller action into play. It is clearly visible that residual captures the variation of friction (variation of mass) while controller remains effective or otherwise.

The experiments involve only non-destructive procedures so that there is no degradation (wear) of the surfaces. In other words, $\mu$ is assumed constant. Experiments involve variation of suspended load mass $M$ in a uniform way till the limit $M_{\text {fail }}$, is reached. $M(t)$ is treated as system parameter under degradation. The experiments were conducted in two distinct phases:

- Offline: In this phase, multiple tests were done with the load being added uniformly. As explained in Section 2.2, variations of $M(t)$ were obtained from the evolution of $r_{2, n}(t)$ found in (57). This provided the time dependent DM of the system parameter $M(t)$.

- Online health monitoring: The maximum limit of additive load mass $M_{\text {fail }}$ was pre-decided keeping in mind the safety of the system. Load was varied until $M_{\text {fail }}$; this was performed in the similar environment as of the offline phase. In real time, estimation of $M(t)$ and associated DPPs, and subsequent RUL predictions were obtained.

\subsection{Case I :Linear variation of mass}

Linear degradation models are frequently employed where incipient degradation does not accelerate subsequent degradation. Here, such a scenario was created through experiments and tested in real time.

\subsubsection{Degradation Test and Degradation model}

Load is varied linearly. Ten experiments are carried out wherein; sand is poured with same environmental conditions to maintain the uniformity. Fig. 18 shows the experimental data and the data mean found at each instant. A linear fit over data mean is obtained using linear regression. The DM can be expressed as,

$$
\begin{aligned}
M(t) & =g_{2}\left(\gamma_{2}, t\right)+v_{M 2}(t) \\
& =\gamma_{2} \times t+v_{M 2}(t)
\end{aligned}
$$

where $g_{2}($.$) is the DM, DPP vector \gamma^{d}=\left\{\gamma^{d}\right\}=\gamma_{2}$ and $v_{M 2}(t) \sim \mathcal{N}\left(0, \sigma_{v_{M 2}}^{2}\right)$. An approximate $\gamma_{2}^{*}=0.005 \mathrm{Kg} / \mathrm{s}$ is obtained. Sum of squared errors provide an approximate standard deviation for process noise $v_{M}, \sigma_{v_{M 2}}=1 \times 10^{-3} \mathrm{Kg}$.

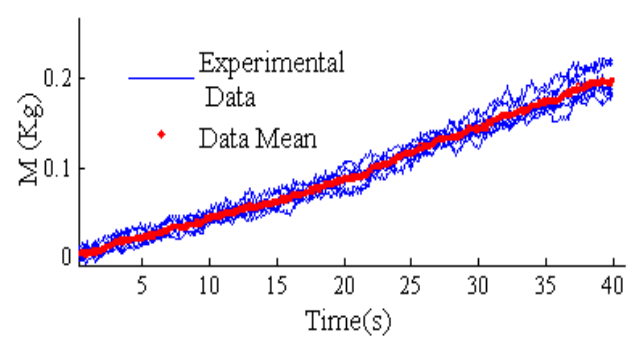

Fig. 18 Degradation Test Data (linear variation)

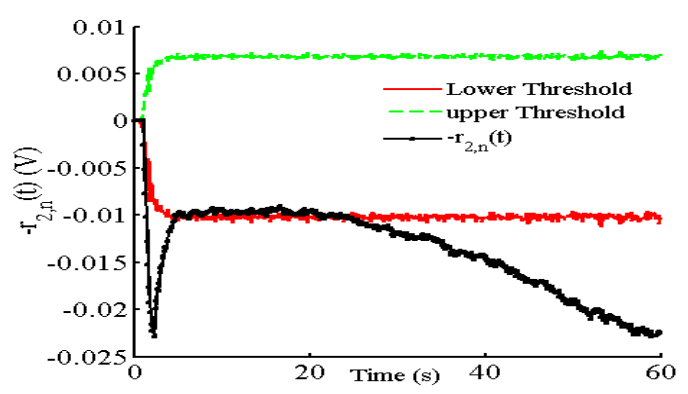

Fig.19 Nominal residual $-r_{2, n}(t)$ while system is under degradation. (linear variation of mass)

\subsubsection{Fault model}

The tuple $\left(M(t), \gamma_{2}, g_{2}\right)$ is formulated in state space as in $(60)$, with $\xi_{2, k} \sim \mathcal{N}\left(0, \sigma_{\xi_{2}}^{2}\right)$ as the additive random walk noise. 


$$
\begin{aligned}
& M_{k}=M_{k-1}+\gamma_{2, k-1} \times \Delta t+v_{M 2_{k-1}} \\
& \gamma_{2, k}=\gamma_{2, k}+\xi_{2, k}
\end{aligned}
$$
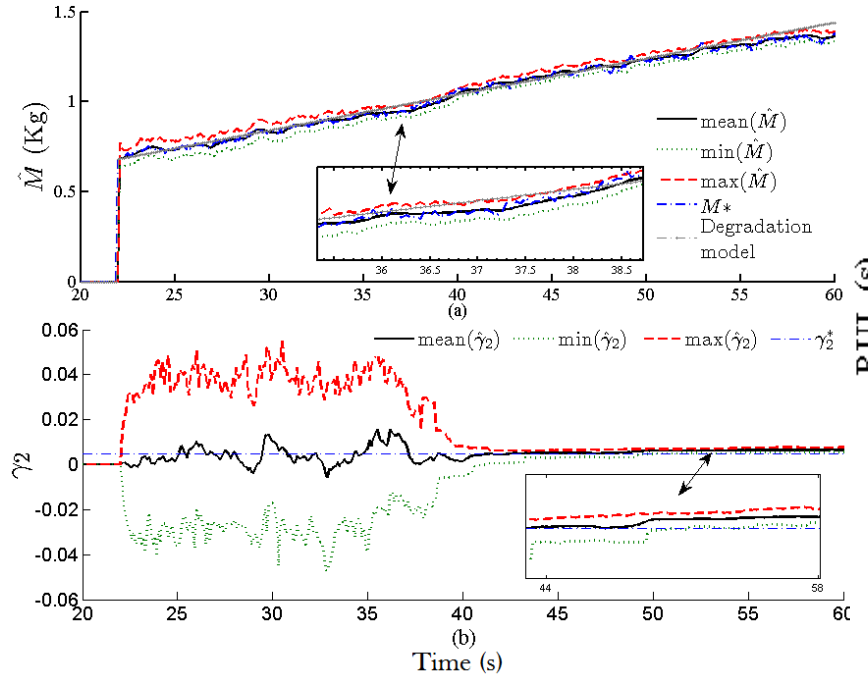

Fig. 20. (a). Estimation of $M$ (b) Estimation of $\gamma_{2}$

$$
\begin{aligned}
& 0=r_{2, n}(t)+\left(M(t)-M_{n}\right) \cdot \frac{\partial\left(r_{2, n}(t)\right)}{\partial(M)} \\
& y_{2, k}=-r_{2, n, k}+w_{2, k}(t)=\left(M_{k}-M_{n}\right)\left(-\frac{\left.\mu_{n} g r_{M d} \operatorname{sgn}\left(\omega_{M d, k}\right)\right)}{k_{b e l t}}\right)+w_{2, k}
\end{aligned}
$$
dation.

\subsubsection{Estimation} with $R M S E_{M}=3.98 \%$.

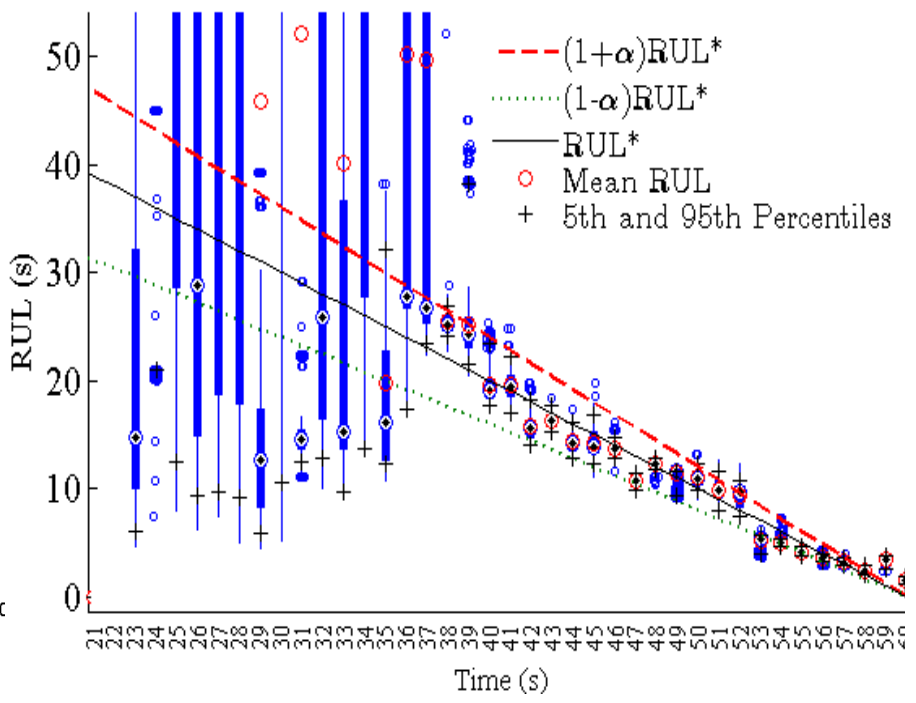

Fig 21: RUL prediction in experimental case: Linear varia-

Observation equation is obtained from the nominal part of $\operatorname{I-ARR}[\underline{R}, \bar{R}]_{2}, r_{2, n}(t)$, as shown in (61) and (62) with $w_{2, k} \sim \mathcal{N}\left(0, \sigma_{w 2}^{2}\right) \cdot \sigma_{w 2}$ is determined from $r_{2, n}(t)$ values during degradation tests of Fig. 18.

For the experiment, load is varied until $M(t)=M_{\text {fail }}=1.5 \mathrm{Kg}$. Fig. 19 shows the nominal residual profile under degra-

The prognostics module is triggered at $t=22 \mathrm{~s}$; estimation and predictions are performed with $N=50$ particles, $\Delta t=0.1 \mathrm{~s}$, $\sigma_{\xi_{2}, k=0}^{2}=1 \times 10^{-6}, \sigma_{v_{M 2}}=1 \times 10^{-3} \mathrm{Kg}, \sigma_{w_{2}}=5 \times 10^{-3} \mathrm{~V}$. For estimation, particle filter assumes measurement noise variance 9 times that of measurement variance $\sigma_{w_{2}}^{2}$ to counter sample impoverishment problem. Estimation of $M$ is shown in Fig. 20a. The true $M^{*}$ is the residual based measurement of $M(t)$ (as described in section 2.2, cf. (15)). State is estimated very accurately

\section{tion of mass}

Estimation of DPP $\gamma_{2}$ is shown in Fig. 20b. Here, reference RMAD is set as $v^{\xi_{2}{ }^{*}}=5 \%$, proportional gain $P_{2}=0.007$, true DPP interval $\left[\gamma_{2, l}^{*}, \gamma_{2, u}^{*}\right]=\left[3 \times 10^{-3}, 7 \times 10^{-3}\right] \mathrm{Kg} / \mathrm{s}$ around the approximately true $\gamma_{2}^{*}=0.005 \mathrm{Kg} / \mathrm{s}$. It should be noted that in the real experiment, $\gamma_{2}^{*}$ is not guaranteed to remain constant; the DM provides only an approximate idea of its magnitude order. Fig. 20b shows the estimation with large initial variance. The estimation spread is reduced effectively from $t=40 \mathrm{~s}$. Thereafter, the estimation mean remains around $\gamma_{2}^{*}$ with RMAD of $6 \%$.

\subsubsection{RUL prediction}


Prediction of RUL is shown in Fig. 21 with $\alpha=0.2$ and $\beta=0.5$.The initial predictions have a very large spread due to the large corresponding spread in $\hat{\gamma}_{2}$. However, after $t=35 \mathrm{~s}$, the RUL is within the $(1 \pm \alpha)$ RUL ${ }^{*}$ bounds with $\overline{R A}=98.64 \%, \overline{R M A D}_{R U L}=9.4 \%$. During the last 3 seconds of experimentation, the sand inflow is stopped gradually (and not abruptly) bringing in certain non-uniformity. As such, RUL predictions at $t=58 \mathrm{~s}, 59 \mathrm{~s}$ and $60 \mathrm{~s}$, do not fall under the $(1 \pm \alpha)$ RUL $^{*}$ bounds that are based upon the ideal linear degradation model.

\subsection{Case II :Exponential variation of mass}

Load is varied in an exponentially. Eight experiments are carried out in total. The considered DM is given in (63) where $g_{3}($.$) is the DM, \theta^{d}=M(t)$, DPP vector $\gamma^{d}=\left\{\gamma^{d}\right\}=\gamma_{3}$ and $v_{M 3}(t) \sim \mathcal{N}\left(0, \sigma_{v_{M 3}}^{2}\right)$.

$$
\begin{aligned}
b_{M d}(t) & =g_{3}\left(M, \gamma_{3}\right)+v_{M 3} \\
& =M_{n} e^{\gamma_{3}(t)}+v_{M 3}
\end{aligned}
$$

Fig. 22a shows the experimental data. Fig. 22b shows the exponential fit over the experimental data mean from which the approximate value of DPP $\gamma_{3}^{*}=0.05 \mathrm{Kg} / \mathrm{s}$, is obtained. Regression residuals provide $\sigma_{v_{M 3}}=8 \times 10^{-4} \mathrm{Kg}$.

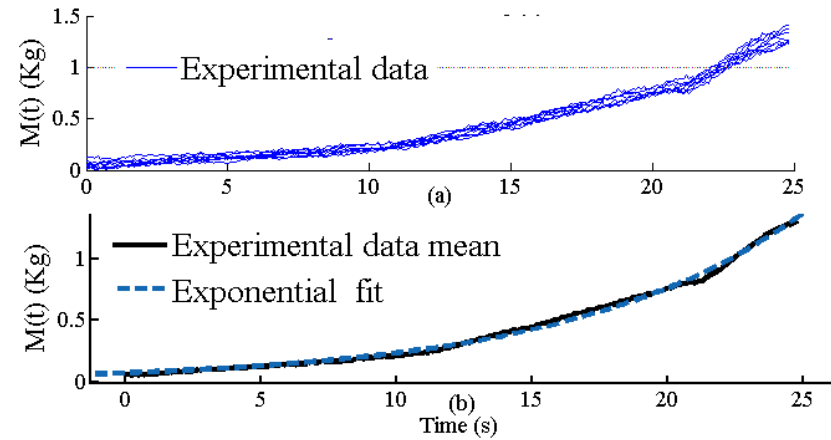

Fig. 22 Exponential variation of mass. (a) experimental data (b). Exponential fit over experimental data mean

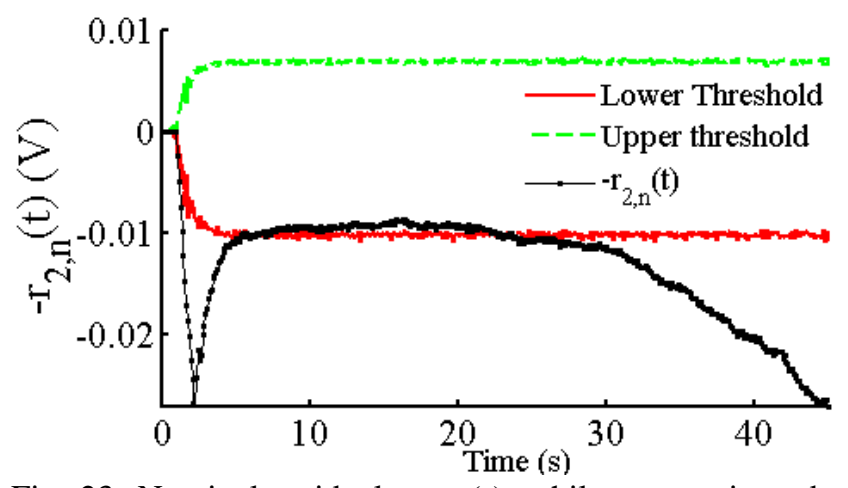

Fig. 23. Nominal residual $-r_{2, n}(t)$ while system is under degradation (exponential case)

\subsubsection{Fault Model}

where $\xi_{3, k} \sim \mathcal{N}\left(0, \sigma_{\xi_{3}}^{2}\right), w_{3, k} \sim \mathcal{N}\left(0, \sigma_{w 3}^{2}\right)$ and the approximation of $\sigma_{w 3}$ is determined from $r_{2, n}(t)$ values during degradation tests. The structure of the observation equation remains same as in (62). For the experiment, load mass is varied until $M(t)=M_{\text {fail }}=1.8 \mathrm{Kg}$. Fig 23 shows the profile of nominal residual under exponential degradation.

\subsubsection{Estimation and RUL Prediction}

The prognostic module is triggered at $t=22 \mathrm{~s}$. It is performed with $\mathrm{N}=50, \Delta t=0.1 \mathrm{~s}, \sigma_{\xi_{3}, k=0}^{2}=4 \times 10^{-6}$ and $\sigma_{w 3}=5 \times 10^{-3} \mathrm{~V}$.

For estimation, particle filter assumes measurement noise variance 9 times that of measurement variance $\sigma_{w 3}^{2}$ to counter 
rately with $R M S E_{M}=3.78 \%$. Fig. $24 \mathrm{~b}$ shows the DPP $\gamma_{3}$ estimation with reference RMAD set as $v^{\xi_{3}{ }^{*}}=10 \%$, proportional gain $P_{3}=0.003$, true DPP interval $\left[\gamma_{3, l}^{*}, \gamma_{3, u}^{*}\right]=\left[1 \times 10^{-2}, 9 \times 10^{-2}\right] \mathrm{Kg} / \mathrm{s}$. Estimation is achieved with $R M S E_{\gamma 3}=7.6 \%$. It must be noted that in reality, $\gamma_{3}^{*}$ cannot be claimed to be the accurate true value of $\gamma_{3}$. Fig. 24c shows the RUL prediction with $\alpha=0.2, \beta=0.5$. Ignoring the initial predictions until $t=32$ (due to large spread), $\overline{R M A D}_{R U L}=9.4 \%$ and $\overline{R A}=97.02 \%$. In fact, the EOL is achieved slightly before than that predicted by DM.

It should be noted that $R M S E_{\gamma}$ in real time experiments is higher than that obtained in simulations as $\gamma^{*}$ does not remaining perfectly constant in real cases. Also, usage of lesser number of particles leads to worse estimation performance. However, overall prediction and estimation performances are very good and satisfactory.
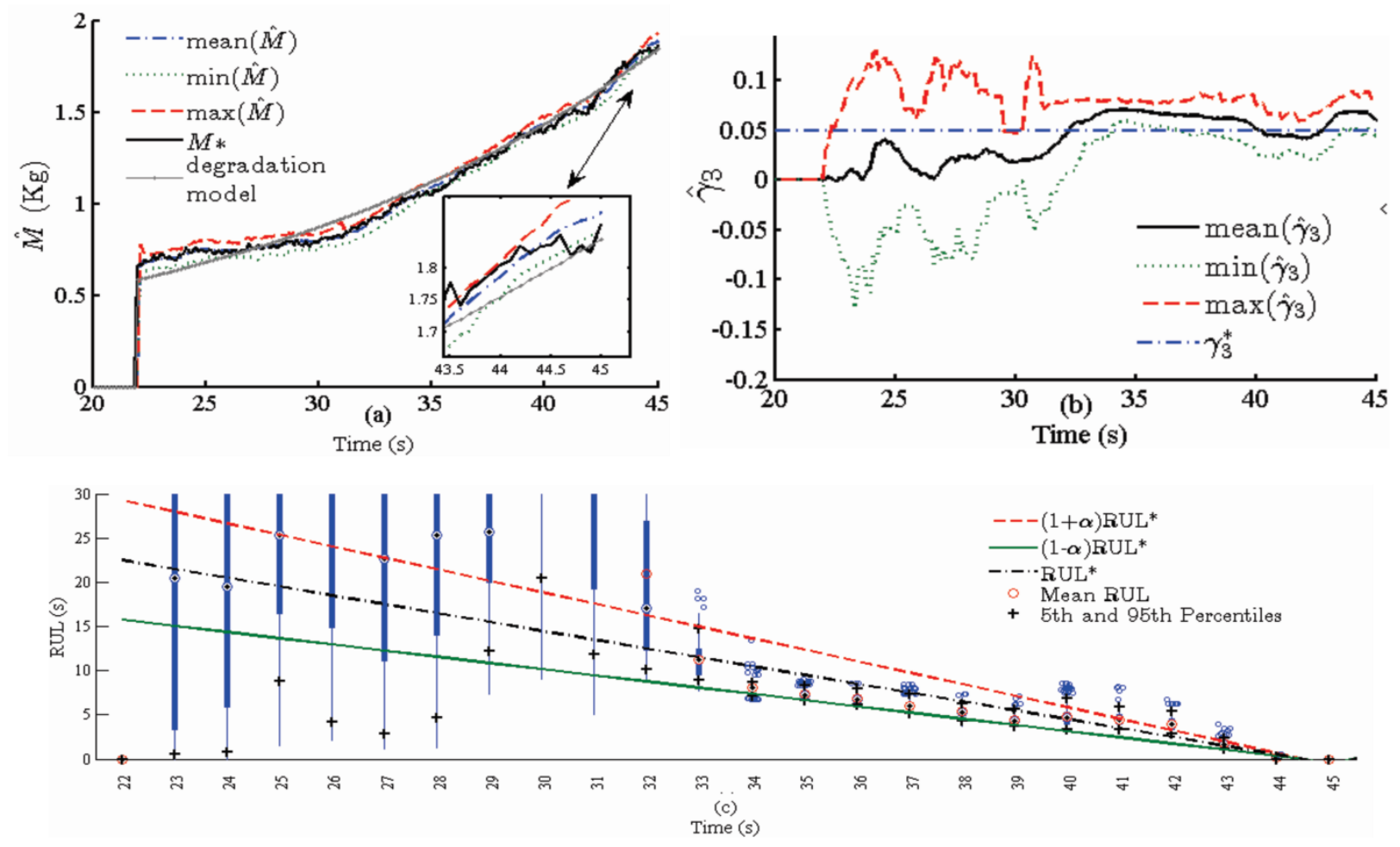

Fig. 24. (a) State estimation for Trail 1 (b) Estimation of DPP $\gamma_{3}$ (c) RUL prediction for case II

\section{Conclusions}

It has been successfully demonstrated through simulation and experimental studies that under single degradation hypothesis, the nominal part of Interval Valued Analytical Redundancy Relations (I-ARRs) derived from the Bond Graph (BG) model of the uncertain system can be used for detection of system parameter's degradation. Subsequent estimation of the state of health and associated degradation progression parameter(s), and prediction of the remaining useful life of the prognostic candidate can be obtained using particle filtering algorithms. This leads to an efficient integration of the benefits of BG modeling framework and Monte Carlo framework. The uncertain part of the I-ARRs is used for robust threshold generation over the nominal part. This enables efficient detection of the degradation commencement, robust to parametric uncertainty. Further, the same nominal residual can be used for obtaining the measurements of state variables in the fault model while the observation equation is developed from the nominal part of the I-ARR. For the latter, a novel algebraic approach is proposed so that the robust detection of degradation and further estimation of state variables of the fault model can be achieved using the same nominal residual in a unified framework. Moreover, this methodology can be extended in presence of multiple degradations which forms a potential future work. In future, the work will be effectively explored for large systems with multiple prognostic candidates. Being sensitive to the control inputs, nominal residual is 
able to capture the parametric degradation profile even while the system outputs remain in feedback closed loop regime. This makes the approach effective for system level health management. Approximation of the distribution of noise present in residuals can be difficult or impossible, due to presence of derivative or integral terms in the arguments. As such, employed Particle filter algorithms form the best choice in this regard, supporting non-Gaussian noises. The novel variance adaptation scheme leads to very good estimation results and involves less complexity in terms of tuning of the involved factors. In future, the latter will be developed further and exploited for similar purposes. Through simulations, this approach has the capability of generating long term and very long term predictions.

Through experiments, capability of obtaining RUL predictions in real time has been shown, although, in very short time window. The associated computational complexity prevents the long and very long-term RUL predictions in real time.

In future, additional ways to obtain the same in sliding time windows will be explored. The method will be extended to achieve very long term predictions in multiple stages, comprising of small time windows, in real time. Although, robustness of the methodology has not been analyzed quantitatively, a qualitative analysis has been presented which helps in an efficient tuning of the PF parameters. As this work forms an effective initial step towards prognostics in BG framework, the same methodology will be applied over complex non-linear thermochemical-hydraulic systems such as fuel cells and vapor generator systems.

\section{Acknowledgements}

The first author is thankful to Prof. Genevieve Dauphin-Tanguy and Ecole Centrale de Lille, for funding the research work.

\section{Appendix A}

Given a real function $f$ of real variables $x=\left[x_{1}, x_{2}, \ldots x_{n}\right]^{T}$ belonging to intervals $X=\left[X_{1}, X_{2}, \ldots X_{n}\right]^{T}[81]$ :

Definition A.1: The interval extension function (IEF), $F(X)$, is any interval valued function that satisfies $F\left(x_{1}, x_{2} \ldots \mathrm{x}_{n}\right)=f\left(x_{1}, x_{2} \ldots \mathrm{x}_{n}\right)$. For degenerate interval arguments, the result must be the degenerate interval $\left[f\left(x_{1}, x_{2} \ldots \mathrm{x}_{n}\right), f\left(x_{1}, x_{2} \ldots \mathrm{x}_{n}\right)\right]$.

Definition A.2: Natural interval extension (NIE) $F$, of $f$ is obtained, by replacing the real arguments with interval arguments and real operators (arithmetic etc. ) by their equivalent interval operators, in the syntactic expression of the real function $f$.

Definition A.3: We say that is $F=F\left(X_{1}, X_{2}, \ldots . X_{n}\right)$ inclusion isotonic if $Y_{i} \subseteq X_{i} \forall i=1,2 . . n \quad \Rightarrow$ $F\left(Y_{1}, Y_{2} . . Y_{n}\right) \subseteq F\left(X_{1}, X_{2} \ldots X_{n}\right)$.

Definition A.4: A rational interval function is an interval-valued function whose values are defined by a specific finite sequence of interval arithmetic operations.

Lemma A.3.1: All rational interval functions are inclusion isotonic.

Theorem A.1 (Fundamental Theorem of Interval Analysis): If $F$ is an inclusion isotonic interval extension of $f$, then $f\left(X_{1}, X_{2} \ldots X_{n}\right) \subseteq F\left(X_{1}, X_{2} \ldots X_{n}\right)$.

Corollary A.1.1: If $F$ is a rational interval function and an interval extension of $f$, then $f\left(X_{1}, X_{2} \ldots X_{n}\right) \subseteq F\left(X_{1}, X_{2} \ldots X_{n}\right)$.

\section{References}

[1] A.K. Jardine, D. Lin, D. Banjevic, A review on machinery diagnostics and prognostics implementing condition-based maintenance, Mechanical systems and signal processing, 20 (2006) 1483-1510. 
[2] J. Lee, F. Wu, W. Zhao, M. Ghaffari, L. Liao, D. Siegel, Prognostics and health management design for rotary machinery systems-Reviews, methodology and applications, Mechanical Systems and Signal Processing, 42 (2014) 314-334.

[3] J. Sikorska, M. Hodkiewicz, L. Ma, Prognostic modelling options for remaining useful life estimation by industry, Mechanical Systems and Signal Processing, 25 (2011) 1803-1836.

[4] A. Heng, S. Zhang, A.C.C. Tan, J. Mathew, Rotating machinery prognostics: State of the art, challenges and opportunities, Mechanical Systems and Signal Processing, 23 (2009) 724-739.

[5] J. Sun, H. Zuo, W. Wang, M.G. Pecht, Application of a state space modeling technique to system prognostics based on a health index for condition-based maintenance, Mechanical Systems and Signal Processing, 28 (2012) 585-596.

[6] D. Louit, R. Pascual, D. Banjevic, A.K.S. Jardine, Condition-based spares ordering for critical components, Mechanical Systems and Signal Processing, 25 (2011) 1837-1848.

[7] N. Gebraeel, J. Pan, Prognostic degradation models for computing and updating residual life distributions in a time-varying environment, Reliability, IEEE Transactions on, 57 (2008) 539-550.

[8] H. Guo, H. Liao, Practical Approaches for Reliability Evaluation Using Degradation Data, Annual Reliability and Maintainability Symposium, 2015.

[9] M. Jouin, R. Gouriveau, D. Hissel, M.-C. Péra, N. Zerhouni, Prognostics of PEM fuel cell in a particle filtering framework, International Journal of Hydrogen Energy, 39 (2014) 481-494.

[10] B. Saha, K. Goebel, J. Christophersen, Comparison of prognostic algorithms for estimating remaining useful life of batteries, Transactions of the Institute of Measurement and Control, (2009).

[11] J.-C. Lu, J. Park, Q. Yang, Statistical inference of a time-to-failure distribution derived from linear degradation data, Technometrics, 39 (1997) 391-400.

[12] X.-S. Si, W. Wang, C.-H. Hu, M.-Y. Chen, D.-H. Zhou, A Wiener-process-based degradation model with a recursive filter algorithm for remaining useful life estimation, Mechanical Systems and Signal Processing, 35 (2013) 219-237.

[13] G. Vachtsevanos, F. Lewis, M. Roemer, A. Hess, B. Wu, Intelligent Fault Diagnosis and Prognosis for Engineering Systems, John Wiley \& Sons, Inc., New Jersey, 2007.

[14] D. Chelidze, J.P. Cusumano, A dynamical systems approach to failure prognosis, Journal of Vibration and Acoustics, 126 (2004) 2-8.

[15] M.S. Kan, A.C.C. Tan, J. Mathew, A review on prognostic techniques for non-stationary and non-linear rotating systems, Mechanical Systems and Signal Processing, 62-63 (2015) 1-20.

[16] M. Schwabacher, A survey of data-driven prognostics, Proceedings of the AIAA Infotech@ Aerospace Conference, 2005, pp. 1-5.

[17] R.K. Neerukatti, K.C. Liu, N. Kovvali, A. Chattopadhyay, Fatigue life prediction using hybrid prognosis for structural health monitoring, Journal of Aerospace Information Systems, 11 (2014) 211-232.

[18] M. Daigle, K. Goebel, Model-based prognostics under limited sensing, Aerospace Conference, 2010 IEEE, IEEE, 2010, pp. 1-12.

[19] M.J. Daigle, K. Goebel, A Model-Based Prognostics Approach Applied to Pneumatic Valves, International Journal of Prognostics and Health Management, 2 (2011).

[20] M.J. Daigle, K. Goebel, Model-based prognostics with concurrent damage progression processes, Systems, Man, and Cybernetics: Systems, IEEE Transactions on, 43 (2013) 535-546.

[21] I. Roychoudhury, M. Daigle, An integrated model-based diagnostic and prognostic framework, Proceedings of the 22nd International Workshop on Principle of Diagnosis (DX'11). Murnau, Germany, 2011.

[22] M. Daigle, B. Saha, K. Goebel, A comparison of filter-based approaches for model-based prognostics, Aerospace Conference, 2012 IEEE, IEEE, 2012, pp. 1-10.

[23] J. Celaya, C. Kulkarni, G. Biswas, S. Saha, K. Goebel, A model-based prognostics methodology for electrolytic capacitors based on electrical overstress accelerated aging, (2011).

[24] C.K.R. Lim, D. Mba, Switching Kalman filter for failure prognostic, Mechanical Systems and Signal Processing, 52-53 (2015) 426-435.

[25] G.L. Plett, Extended Kalman filtering for battery management systems of LiPB-based HEV battery packs: Part 3. State and parameter estimation, Journal of Power sources, 134 (2004) 277-292.

[26] M.J. Daigle, A. Bregon, I. Roychoudhury, Distributed prognostics based on structural model decomposition, (2014). 
[27] M. Gašperin, Đ. Juričić, P. Boškoski, J. Vižintin, Model-based prognostics of gear health using stochastic dynamical models, Mechanical Systems and Signal Processing, 25 (2011) 537-548.

[28] D. An, N.H. Kim, J.-H. Choi, Practical options for selecting data-driven or physics-based prognostics algorithms with reviews, Reliability Engineering \& System Safety, 133 (2015) 223-236.

[29] M.S. Arulampalam, S. Maskell, N. Gordon, T. Clapp, A tutorial on particle filters for online nonlinear/nonGaussian Bayesian tracking, Signal Processing, IEEE Transactions on, 50 (2002) 174-188.

[30] M.E. Orchard, A particle filtering-based framework for on-line fault diagnosis and failure prognosis, Ph.D dissertation, Georgia Institute of Technology, 2007.

[31] P. Baraldi, M. Compare, S. Sauco, E. Zio, Ensemble neural network-based particle filtering for prognostics, Mechanical Systems and Signal Processing, 41 (2013) 288-300.

[32] B. Saha, K. Goebel, Modeling Li-ion battery capacity depletion in a particle filtering framework, Proceedings of the annual conference of the prognostics and health management society, 2009, pp. 29092924.

[33] B. Saha, K. Goebel, S. Poll, J. Christophersen, Prognostics methods for battery health monitoring using a Bayesian framework, Instrumentation and Measurement, IEEE Transactions on, 58 (2009) 291-296.

[34] M. Abbas, A.A. Ferri, M.E. Orchard, G.J. Vachtsevanos, An intelligent diagnostic/prognostic framework for automotive electrical systems, Intelligent Vehicles Symposium, 2007 IEEE, IEEE, 2007, pp. 352-357.

[35] E. Bechhoefer, A method for generalized prognostics of a component using Paris law, ANNUAL FORUM PROCEEDINGS-AMERICAN HELICOPTER SOCIETY, AMERICAN HELICOPTER SOCIETY, INC, 2008, pp. 1460.

[36] F. Cadini, E. Zio, D. Avram, Monte Carlo-based filtering for fatigue crack growth estimation, Probabilistic Engineering Mechanics, 24 (2009) 367-373.

[37] E. Zio, G. Peloni, Particle filtering prognostic estimation of the remaining useful life of nonlinear components, Reliability Engineering \& System Safety, 96 (2011) 403-409.

[38] E. Zio, Prognostics and health management of industrial equipment, Diagnostics and Prognostics of Engineering Systems: Methods and Techniques, (2012) 333-356.

[39] M.J. Daigle, K. Goebel, A model-based prognostics approach applied to pneumatic valves, International Journal of Prognostics and Health Management Volume 2 (color), (2011) 84.

[40] P. Baraldi, F. Mangili, E. Zio, Investigation of uncertainty treatment capability of model-based and datadriven prognostic methods using simulated data, Reliability Engineering \& System Safety, 112 (2013) 94-108.

[41] D. An, J.-H. Choi, N.H. Kim, Prognostics 101: A tutorial for particle filter-based prognostics algorithm using Matlab, Reliability Engineering \& System Safety, 115 (2013) 161-169.

[42] M. Orchard, G. Kacprzynski, K. Goebel, B. Saha, G. Vachtsevanos, Advances in uncertainty representation and management for particle filtering applied to prognostics, Prognostics and health management, 2008. phm 2008. international conference on, IEEE, 2008, pp. 1-6.

[43] M. Daigle, K. Goebel, Model-based prognostics with fixed-lag particle filters, Conference of the PHM Society, 2009.

[44] Y. Hu, P. Baraldi, F. Di Maio, E. Zio, A particle filtering and kernel smoothing-based approach for new design component prognostics, Reliability Engineering \& System Safety, 134 (2015) 19-31.

[45] S. Kunche, C. Chen, M. Pecht, A review of PHM system's architectural frameworks, The 54th Meeting of the Society for Machinery Failure Prevention Technology, Dayton, OH, 2012.

[46] R. Moghaddass, M.J. Zuo, An integrated framework for online diagnostic and prognostic health monitoring using a multistate deterioration process, Reliability Engineering \& System Safety, 124 (2014) $92-$ 104.

[47] D.C. Karnopp, D.L. Margolis, R.C. Rosenberg, System Dynamics: Modeling, Simulation, and Control of Mechatronic Systems, Wiley, 2012.

[48] W. Borutzky, Bond Graph Modelling of Engineering Systems, Springer, 2011.

[49] A. Mukherjee, A.K. Samantaray, Bond graph in modeling, simulation and fault identification, IK International Pvt Ltd, 2006.

[50] J. Thoma, B.O. Bouamama, Modelling and simulation in thermal and chemical engineering: A bond graph approach, Springer, 2000.

[51] A.K. Samantaray, B.O. Bouamama, Model-based process supervision, Springer, 2008.

[52] K. Medjaher, A.K. Samantaray, B. Ould Bouamama, M. Staroswiecki, Supervision of an industrial steam generator. Part II: Online implementation, Control Engineering Practice, 14 (2006) 85-96. 
[53] R. Merzouki, K. Medjaher, M.A. Djeziri, B. Ould-Bouamama, Backlash fault detection in mechatronic system, Mechatronics, 17 (2007) 299-310.

[54] R. Loureiro, S. Benmoussa, Y. Touati, R. Merzouki, B. Ould Bouamama, Integration of Fault Diagnosis and Fault-Tolerant Control for Health Monitoring of a Class of MIMO Intelligent Autonomous Vehicles, Vehicular Technology, IEEE Transactions on, 63 (2014) 30-39.

[55] S. Benmoussa, B.O. Bouamama, R. Merzouki, Bond Graph Approach for Plant Fault Detection and Isolation: Application to Intelligent Autonomous Vehicle, Automation Science and Engineering, IEEE Transactions on, 11 (2014) 585-593.

[56] N. Chatti, A. Gehin, B. Ould-Bouamama, R. Merzouki, Functional and Behavior Models for the Supervision of an Intelligent and Autonomous System, Automation Science and Engineering, IEEE Transactions on, 10 (2013) 431-445.

[57] R. El Harabi, B. Ould-Bouamama, M.K.B. Gayed, M.N. Abdelkrim, Pseudo bond graph for fault detection and isolation of an industrial chemical reactor part I: bond graph modeling, Proceedings of the 2010 Spring Simulation Multiconference, Society for Computer Simulation International, 2010, pp. 220.

[58] Y. Ming, W. Danwei, L. Ming, H. Lei, Prognosis of Hybrid Systems With Multiple Incipient Faults: Augmented Global Analytical Redundancy Relations Approach, Systems, Man and Cybernetics, Part A: Systems and Humans, IEEE Transactions on, 41 (2011) 540-551.

[59] B.O. Bouamama, A. Samantaray, M. Staroswiecki, G. Dauphin-Tanguy, Derivation of constraint relations from bond graph models for fault detection and isolation, SIMULATION SERIES, 35 (2003) 104-109.

[60] A.K. Samantaray, B.O. Bouamama, Model-based process supervision: a bond graph approach, Springer Science \& Business Media, 2008.

[61] D. Yang, E. Tarasov, C. Sueur, B. Ould Bouamama, New unknown input observer for control design: a bond graph approach, System, Structure and Control, 2013, pp. 611-616.

[62] G. Dauphin-Tanguy, C.S. Kam, How to model parameter uncertainties in a bond graph framework, Simulation in Industry, 11th European Simulation Symposium, ESS, 1999, pp. 121-125.

[63] C. Sié Kam, G. Dauphin-Tanguy, Bond graph models of structured parameter uncertainties, Journal of the Franklin Institute, 342 (2005) 379-399.

[64] M.A. Djeziri, R. Merzouki, B.O. Bouamama, G. Dauphin-Tanguy, Robust fault diagnosis by using bond graph approach, Mechatronics, IEEE/ASME Transactions on, 12 (2007) 599-611.

[65] M.A. Djeziri, B. Ould Bouamama, R. Merzouki, Modelling and robust FDI of steam generator using uncertain bond graph model, Journal of Process Control, 19 (2009) 149-162.

[66] M.A. Djeziri, B.O. Bouamama, G. Dauphin-Tanguy, R. Merzouki, LFT Bond Graph Model-Based Robust Fault Detection and Isolation, Bond Graph Modelling of Engineering Systems, Springer, 2011, pp. 105-133.

[67] M. Jha, G. Dauphin-Tanguy, B. Ould Bouamama, Robust FDI based on LFT BG and relative activity at junction, Control Conference (ECC), 2014 European, IEEE, 2014, pp. 938-943.

[68] B. Sun, S. Zeng, R. Kang, M.G. Pecht, Benefits and challenges of system prognostics, Reliability, IEEE Transactions on, 61 (2012) 323-335.

[69] K. Medjaher, N. Zerhouni, Hybrid prognostic method applied to mechatronic systems, The International Journal of Advanced Manufacturing Technology, 69 (2013) 823-834.

[70] K. Medjaher, N. Zerhouni, Residual-based failure prognostic in dynamic systems, 7th IFAC International Symposium on Fault Detection, Supervision and Safety of Technical Processes, SAFE PROCESS'09., IFAC, 2009, pp. 6 pages.

[71] M. Djeziri, B. Ananou, M. Ouladsine, Data driven and model based fault prognosis applied to a mechatronic system, Power Engineering, Energy and Electrical Drives (POWERENG), 2013 Fourth International Conference on, IEEE, 2013, pp. 534-539.

[72] M. Djeziri, H. Toubakh, M. Ouladsine, Fault prognosis based on fault reconstruction: Application to a mechatronic system, Systems and Control (ICSC), 2013 3rd International Conference on, IEEE, 2013, pp. 383388.

[73] S. Uckun, K. Goebel, P.J. Lucas, Standardizing research methods for prognostics, Prognostics and Health Management, 2008. PHM 2008. International Conference on, IEEE, 2008, pp. 1-10.

[74] A. Saxena, J. Celaya, B. Saha, S. Saha, K. Goebel, Metrics for offline evaluation of prognostic performance, International Journal of Prognostics and Health Management Volume 1 (color), (2010) 4. 
920 [75] M. Jha, G. Dauphin-Tanguy, B. Ould Bouamama, Integrated Diagnosis and Prognosis of Uncertain 921 Systems: A Bond Graph Approach Second European Conference of the PHM Society 2014 European 922 Conference of the PHM Society 2014 Proceedings, -Nantes France, 2014, pp. 391-400.

923 [76] A.K. Samantaray, K. Medjaher, B. Ould Bouamama, M. Staroswiecki, G. Dauphin-Tanguy, Diagnostic bond 924 graphs for online fault detection and isolation, Simulation Modelling Practice and Theory, 14 (2006) 237-262.

925 [77] M. Staroswiecki, G. Comtet-Varga, Analytical redundancy relations for fault detection and isolation in 926 algebraic dynamic systems, Automatica, 37 (2001) 687-699.

927 [78] W. Borutzky, Failure Prognosis for Hybrid Systems Based on ARR Residuals, Bond Graph Model-based 928 Fault Diagnosis of Hybrid Systems, Springer, 2015, pp. 221-233.

929 [79] S.G. Krantz, H.R. Parks, The implicit function theorem: history, theory, and applications, Springer Science 930 \& Business Media, 2012.

931 [80] R.E. Moore, Methods and applications of interval analysis, SIAM, 1979.

932 [81] R.E. Moore, R.B. Kearfott, M.J. Cloud, Introduction to interval analysis, Siam, 2009.

933 [82] A. Doucet, N. De Freitas, N. Gordon, An introduction to sequential Monte Carlo methods, Springer, 2001.

934 [83] E. Tarasov, C. Sueur, B.O. Bouamama, G. Dauphin-Tanguy, Flat control of a torsion bar with unknown 935 input estimation, Control Applications (CCA), 2014 IEEE Conference on, IEEE, 2014, pp. 2054-2059.

936 [84] C. Kleijn, Torsion Bar 2.0 Reference Manual, Controllab Products B.V., Enschede, 2011.

937 [85] J.F. Broenink, Modelling, simulation and analysis with 20-sim, Journal A, 38 (1997) 22-25.

938 [86] C. Kleijn, Differ H.G., 20-SIM 4C 2.1 Reference Manual, Enschede, Controllab Products B.V., 2013.

939 [87] S.M. Rump, INTLAB-interval laboratory, Springer, 1999.

940 [88] T. Li, S. Sun, T.P. Sattar, J.M. Corchado, Fight sample degeneracy and impoverishment in particle filters: A 941 review of intelligent approaches, Expert Systems with applications, 41 (2014) 3944-3954. 
Table I

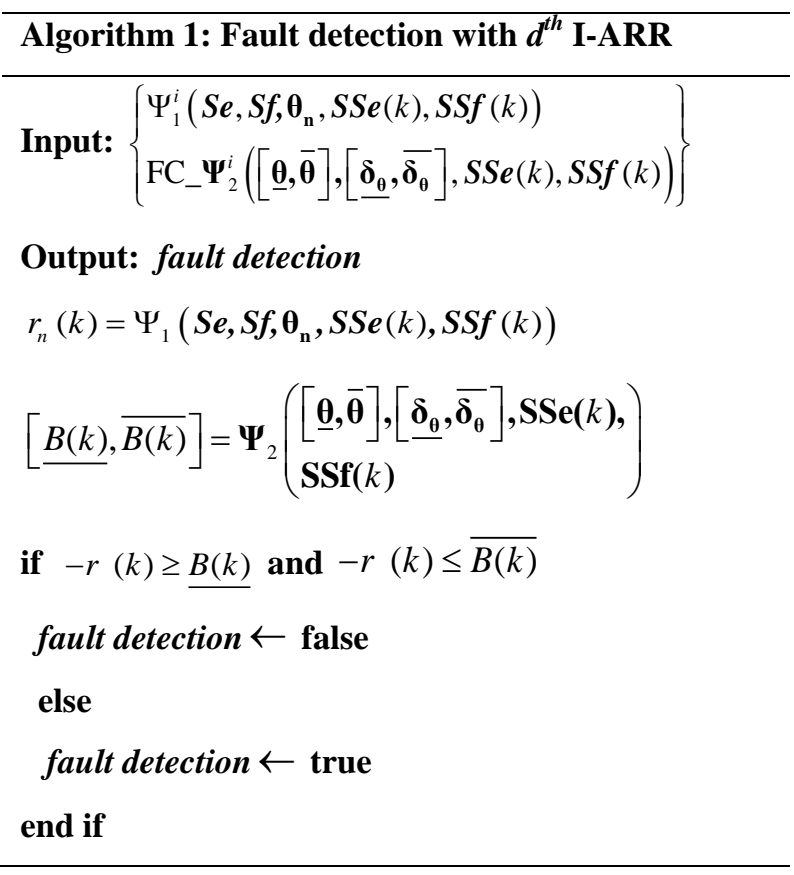

\section{Table II}

\section{Algorithm 2: Estimation using SIR filter}

Inputs: $\left\{\left(\theta_{k-1}^{d, i}, \boldsymbol{\gamma}_{k-1}^{d, i}\right), \mathrm{w}_{k-1}^{i}\right\}_{i=1}^{N}, y_{k}^{d}$

Output: $\left\{\left(\theta_{k}^{d, i}, \boldsymbol{\gamma}_{k}^{d, i}\right), \mathrm{w}_{k}^{i}\right\}_{i=1}^{N}$

for $i=1$ to $\mathrm{N}$ do

$\boldsymbol{\gamma}_{k}^{d, i} \sim p\left(\boldsymbol{\gamma}_{k}^{d, i} \mid \boldsymbol{\gamma}_{k-1}^{d, i}\right)$

$\theta_{k}^{d, i} \sim p\left(\theta_{k}^{d, i} \mid \theta_{k-1}^{d, i}, \gamma_{k-1}^{d, i}\right)$

$\mathrm{w}_{k}^{i} \sim p\left(y_{k}^{d} \mid \theta_{k}^{d, i}, \gamma_{k}^{d, i}\right)$

end for

$W \leftarrow \sum_{i=1}^{N} \mathrm{w}_{k}^{i}$

for $i=1$ to $\mathrm{N}$ do

$\mathrm{w}_{k}^{i} \leftarrow \mathrm{w}_{k}^{i} / W$

end for
Table III

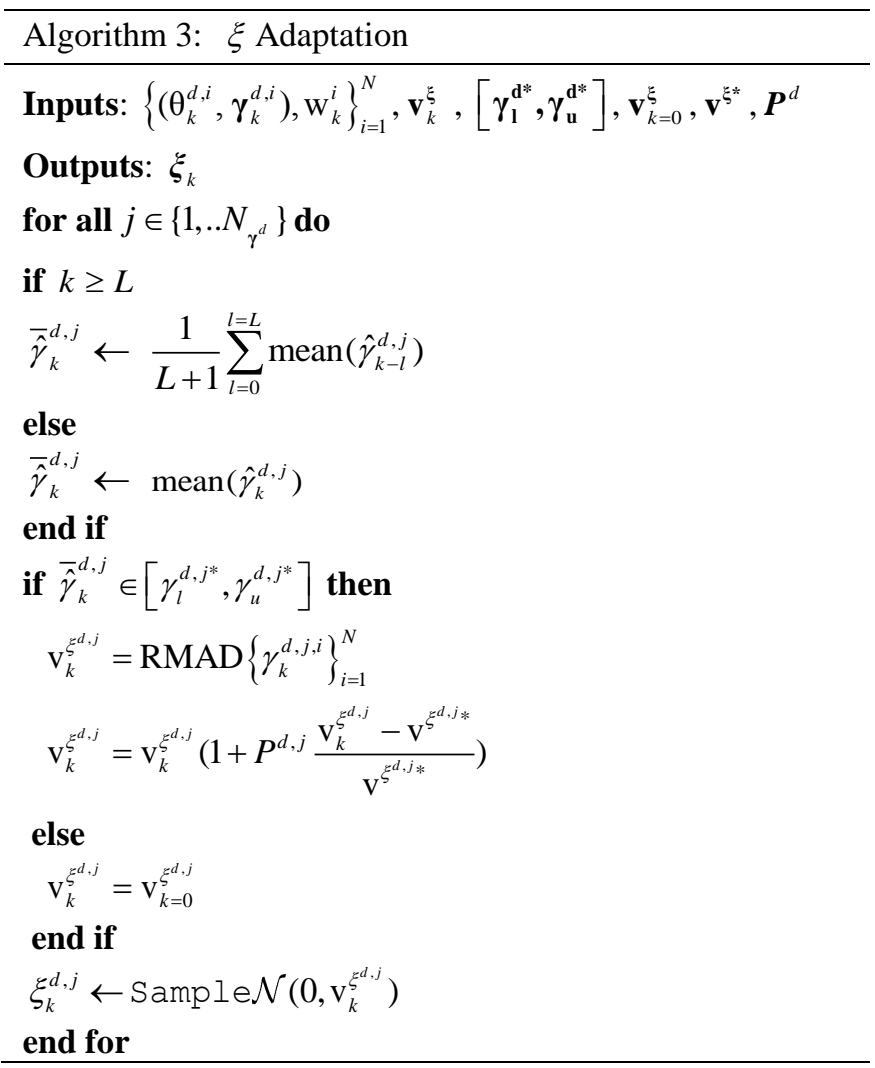

Table IV

\begin{tabular}{l}
\hline Algorithm 4: RUL Prediction \\
\hline Inputs: $\left\{\left(\theta_{k}^{d, i}, \boldsymbol{\gamma}_{k}^{d, i}\right), \mathrm{w}_{k}^{i}\right\}_{i=1}^{N}$ \\
Variable: $l$ \\
Outputs: $\left\{R U L_{k}^{d^{d, i}}, \mathrm{w}_{k}^{i}\right\}_{i=1}^{N}$ \\
$\quad$ for $i=1$ to $N$ do \\
$\quad l=\mathbf{0}$ \\
$\quad$ while $\theta_{k+l}^{d, i} \leq \theta_{f a i l}^{d}$ do \\
$\quad \boldsymbol{\gamma}_{k+1}^{d, i} \sim p\left(\boldsymbol{\gamma}_{k+1}^{d, i} \mid \boldsymbol{\gamma}_{k}^{d, i}\right)$ \\
$\quad \theta_{k+1}^{d, i} \sim p\left(\theta_{k+1}^{d, i} \mid \theta_{k}^{d, i}, \boldsymbol{\gamma}_{k}^{d, i}\right)$ \\
$\quad l \leftarrow l+1$ \\
$\quad$ end while \\
$R U L_{k}^{d, i} \leftarrow l$ \\
end for
\end{tabular}


Table V

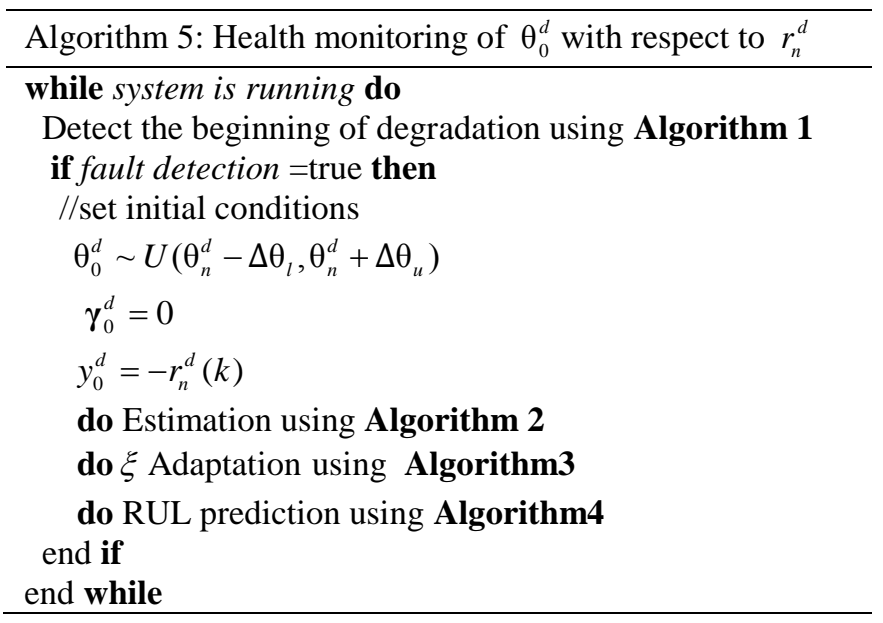

Table VI List of parametric values

\begin{tabular}{lll}
\hline $\begin{array}{c}\text { Parameter } \\
\theta\end{array}$ & \multicolumn{1}{c}{$\begin{array}{c}\text { Nominal Value } \\
\theta_{n}\end{array}$} & $\begin{array}{l}\text { Multiplicative } \\
\text { Uncertainty } \\
{\left[\delta_{\theta}, \overline{\delta_{\theta}}\right]}\end{array}$ \\
\hline$J m$ & $6.76 \times 10^{-6} \mathrm{~kg} \cdot \mathrm{m}^{2} / \mathrm{rad}$ & {$[-0.02,0.02]$} \\
$f_{m}$ & $2 \times 10^{-6} \mathrm{~N} \cdot \mathrm{m} \cdot \mathrm{s} / \mathrm{rad}$ & {$[0,0.3]$} \\
$J_{M d}$ & $9.07 \times 10^{-4} \mathrm{~kg} \cdot \mathrm{m}^{2} / \mathrm{rad}$ & {$[-0.1,0.1]$} \\
$b_{M d}$ & $5.025 \times 10^{-3} \mathrm{~N} \cdot \mathrm{m} \cdot \mathrm{s} / \mathrm{rad}$ & {$[0,0.2]$} \\
$J_{L d}$ & $1.37 \times 10^{-3} \mathrm{~kg} \cdot \mathrm{m}^{2} / \mathrm{rad}$ & {$[-0.1,0.1]$} \\
$b_{L d}$ & $2.5 \times 10^{-5} \mathrm{~N} \cdot \mathrm{m} \cdot \mathrm{s} / \mathrm{rad}$ & {$[0,0.2]$} \\
$k_{s}$ & $1.786 \mathrm{~N} \cdot \mathrm{m} / \mathrm{rad}$ & {$[-0.1,0.1]$} \\
$R_{s}$ & $5.11 \times 10^{-4} \mathrm{~N} \cdot \mathrm{m} / \mathrm{rad}$ & {$[-0.1,0.1]$} \\
$k_{m}$ & $3.89 \times 10^{-4} \mathrm{Nm} / \mathrm{A}$ & - \\
$k_{b e l t}$ & 3.75 & - \\
$L a$ & $1.34 \times 10^{-3} \mathrm{H}$ & - \\
$R a$ & $1.23 \Omega$ & - \\
$\mu$ & 0.27 & {$[-0.1,0.1]$} \\
\hline
\end{tabular}


